\title{
RAD51D Aberrant Splicing in Breast Cancer: Identification of Splicing Regulatory Elements and Minigene-Based Evaluation of 53 DNA Variants
}

\author{
Elena Bueno-Martínez $^{1}{ }^{\mathbb{D}}$, Lara Sanoguera-Miralles ${ }^{1}{ }^{\mathbb{D}}$, Alberto Valenzuela-Palomo ${ }^{1}$, Víctor Lorca $^{2}$, \\ Alicia Gómez-Sanz ${ }^{2}$, Sara Carvalho ${ }^{3}$, Jamie Allen ${ }^{3}$, Mar Infante ${ }^{4} \mathbb{D}$, Pedro Pérez-Segura ${ }^{2}$, Conxi Lázaro ${ }^{5}$, \\ Douglas F. Easton $^{3}$, Peter Devilee ${ }^{6}\left(\mathbb{D}\right.$, Maaike P. G. Vreeswijk ${ }^{6} \mathbb{D}$, Miguel de la Hoya ${ }^{2, *},+(\mathbb{D})$ \\ and Eladio A. Velasco ${ }^{1, *,+}$
}

1 Splicing and Genetic Susceptibility to Cancer Laboratory, Unidad de Excelencia Instituto de Biología y Genética Molecular, Consejo Superior de Investigaciones Científicas (CSIC-UVa), 47003 Valladolid, Spain; elena.bueno@uva.es (E.B.-M.); lara.sanoguera@uva.es (L.S.-M.); alberto.valenzuela@ibgm.uva.es (A.V.-P.)

2 Molecular Oncology Laboratory CIBERONC, IdISSC (Instituto de Investigación Sanitaria del Hospital Clínico San Carlos), Hospital Clinico San Carlos, 28040 Madrid, Spain; victor.lorca@salud.madrid.org (V.L.); agomezsanz@salud.madrid.org (A.G.-S.); pedro.perez@salud.madrid.org (P.P.-S.)

3 Centre for Cancer Genetic Epidemiology, Department of Public Health and Primary Care, University of Cambridge, Cambridge CB1 8RN, UK; sc2017@medschl.cam.ac.uk (S.C.); jma73@medschl.cam.ac.uk (J.A.); dfe20@medschl.cam.ac.uk (D.F.E.)

check for

updates

Citation: Bueno-Martínez, E.; Sanoguera-Miralles, L.; ValenzuelaPalomo, A.; Lorca, V.; Gómez-Sanz,

A.; Carvalho, S.; Allen, J.; Infante, M.; Pérez-Segura, P.; Lázaro, C.; et al. RAD51D Aberrant Splicing in Breast Cancer: Identification of Splicing Regulatory Elements and MinigeneBased Evaluation of 53 DNA Variants. Cancers 2021, 13, 2845. https:// doi.org/10.3390/cancers13112845

Academic Editor: Ana Vega

Received: 16 April 2021

Accepted: 3 June 2021

Published: 7 June 2021

Publisher's Note: MDPI stays neutral with regard to jurisdictional claims in published maps and institutional affiliations.

Copyright: (C) 2021 by the authors. Licensee MDPI, Basel, Switzerland. This article is an open access article distributed under the terms and conditions of the Creative Commons Attribution (CC BY) license (https:/ / creativecommons.org/licenses/by/ $4.0 /)$.
4 Cancer Genetics, Unidad de Excelencia Instituto de Biología y Genética Molecular (CSIC-UVa), 47003 Valladolid, Spain; minfante@ibgm.uva.es

5 Hereditary Cancer Program, Catalan Institute of Oncology, IDIBELL and CIBERONC, 08908 Hospitalet de Llobregat, Spain; clazaro@iconcologia.net

6 Department of Human Genetics, Leiden University Medical Center, 2300RC Leiden, The Netherlands; P.Devilee@lumc.nl (P.D.); M.P.G.Vreeswijk@lumc.nl (M.P.G.V.)

* Correspondence: miguel.hoya@salud.madrid.org (M.d.1.H.); eavelsam@ibgm.uva.es (E.A.V.)

$+\quad$ These authors are joint senior authors.

Simple Summary: In the BRIDGES project, the breast/ovarian cancer gene RAD51D has been sequenced in $>113,000$ women. In the present study, we focused on the impact that 11 pre-selected $R A D 51 D$ variants at the intron/exon boundaries had on the splicing process (intron removal). For this purpose, we developed a splicing reporter minigene, containing $R A D 51 D$-exons 2-9 wherein any variant could be introduced and functionally assayed for splicing alterations. All variants impaired splicing, 10 of which caused complete splicing aberrations. Moreover, we developed a minigene-based strategy to search for non-canonical, spliceogenic variants that disrupted splicing enhancers/silencers in the non-constitutive exon 3. Twenty-six BRIDGES and 16 artificial exon 3 variants were also tested. Thirty variants impaired splicing by producing variable amounts of the FL transcript. In total, up to 9 variants were classified as Likely Pathogenic, and therefore were clinically actionable. Carriers may benefit from tailored prevention protocols and therapies.

Abstract: $R A D 51 D$ loss-of-function variants increase lifetime risk of breast and ovarian cancer. Splicing disruption is a frequent pathogenic mechanism associated with variants in susceptibility genes. Herein, we have assessed the splicing and clinical impact of splice-site and exonic splicing enhancer (ESE) variants identified through the study of $\sim 113,000$ women of the BRIDGES cohort. A RAD51D minigene with exons 2-9 was constructed in splicing vector pSAD. Eleven BRIDGES splice-site variants (selected by MaxEntScan) were introduced into the minigene by site-directed mutagenesis and tested in MCF-7 cells. The 11 variants disrupted splicing, collectively generating 25 different aberrant transcripts. All variants but one produced negligible levels $(<3.4 \%)$ of the full-length (FL) transcript. In addition, ESE elements of the alternative exon 3 were mapped by testing four overlapping exonic microdeletions ( $\geq 30-b p)$, revealing an ESE-rich interval (c.202_235del) with critical sequences for exon 3 recognition that might have been affected by germline variants. Next, 26 BRIDGES variants and 16 artificial exon 3 single-nucleotide substitutions were also assayed. Thirty variants impaired splicing with variable amounts (0-65.1\%) of the FL transcript, although only 
c.202G > A demonstrated a complete aberrant splicing pattern without the FL transcript. On the other hand, c.214T $>C$ increased efficiency of exon 3 recognition, so only the FL transcript was detected (100\%). In conclusion, 41 RAD51D spliceogenic variants (28 of which were from the BRIDGES cohort) were identified by minigene assays. We show that minigene-based mapping of ESEs is a powerful approach for identifying ESE hotspots and ESE-disrupting variants. Finally, we have classified nine variants as likely pathogenic according to ACMG/AMP-based guidelines, highlighting the complex relationship between splicing alterations and variant interpretation.

Keywords: breast cancer; ovarian cancer; susceptibility genes; RAD51D; ESE; ESS; aberrant splicing; VUS; minigene; clinical interpretation

\section{Introduction}

$R A D 51 D$ [MIM\#602954] is one of the five RAD51 paralogs ( $R A D 51 B, R A D 51 C, R A D 51 D$, $X R C C 2, X R C C 3)$ that play an important role in the repair of DNA double-strand breaks via homologous recombination [1,2]. RAD51D loss-of-function variants confer risk of breast and/or ovarian cancer [3,4]. In a cohort of 6690 families, RAD51D pathogenic variants were associated with relative risks of 7.6 and 1.83 for tubo-ovarian cancer and breast cancer, respectively [3]. Recently, two large-scale studies have estimated an overall breast cancer relative risk of 1.8 and 1.72 , respectively $[5,6]$. The association with triple-negative (TN) breast cancer $(R R=6.1)$ appears to be particularly strong [5].

During RNA splicing, introns from eukaryotic genes are removed from pre-mRNA, and consecutive exons are precisely joined together to form mature mRNA [7]. Exon recognition is a crucial step controlled by a large number of trans-acting factors, including ribonucleoproteins and splicing factors as well as cis-acting sequences identified by splicing machinery. The array of splicing signals is extensive and includes $5^{\prime}$ and $3^{\prime}$ splice sites, the polypyrimidine tract, the branch point and exonic and intronic splicing enhancers (ESE/ISE) and silencers (ESS/ISS) that stimulate or repress exon inclusion in mature mRNA [8]. It has been proposed that $\sim 60 \%$ of disease-causing variants disrupt pre-mRNA processing [9], generating aberrant transcripts that can affect protein function and correlate with an increased risk of a given genetic disease. Consequently, it is imperative to assess the biological and clinical significance of spliceogenic variants in order to improve genetic diagnosis and counseling as well as to trigger new therapeutic interventions [10]. Unfortunately, the lack of accurate in silico predictors makes functional assays vital to doing so. Next, splicing functional assays, either from patient or minigene RNAs, provide critical data to evaluate the pathogenicity of a particular genetic variant [11-15].

Our study was conducted using the framework of the BRIDGES project (Breast Cancer After Diagnostic Gene Sequencing; https: / /bridges-research.eu/, accessed on 1 April 2021), which has sequenced a panel of 34 known or suspected breast cancer susceptibility genes in 60,466 cases and 53,461 controls [5]. We bioinformatically analyzed $47 R A D 51 D$ variants from the intron/exon boundaries, 11 of which were selected and functionally tested in a RAD51D-splicing reporter minigene including exons 2-9 (mgR51D_ex2-9). In addition, we functionally mapped ESE-rich regions of the non-constitutive exon 3 by overlapping microdeletions and analyzed 42 candidate BRIDGES and artificial ESE variants. Finally, we made a tentative clinical classification of BRIDGES variants according to ACMG/AMP (American College of Medical Genetics and Genomics and the Association for Molecular Pathology)-based guidelines.

\section{Materials and Methods}

Ethical approval for this study was obtained from the Ethics Committee of the Spanish National Research Council-CSIC (28 May 2018). 


\subsection{Variant and Transcript Annotations}

Forty-seven variants from the intron-exon boundaries ( $3^{\prime}$ splice-site: intron/exon [IVS-10_IVS-1/2nt]; $5^{\prime}$ splice-site: exon/intron [2nt/IVS+1_IVS+10]) [16] and 34 from RAD51D exon 3 were collected from the BRIDGES sequencing data [5]. RNA outcomes and predicted protein products were described according to the Human Genome Variation Society guidelines (http:/ / varnomen.hgvs.org/, accessed on 1 April 2021) using Ensembl reference transcript ID ENST00000345365.10 (GenBank NM_002878.3). To simplify, we also annotated splicing events, as previously described [17].

\subsection{Bioinformatics}

The workflow of the splicing assays is outlined in Figure S1. To identify potential splicing variants, in silico studies were performed using MaxEntScan (MES, http: //hollywood.mit.edu/burgelab/maxent/Xmaxentscan_scoreseq.html, accessed on 1 April 2021) (cut-off $\geq 3.0$ ) [18] and NNSplice (https:/ / www.fruitfly.org/seq_tools/splice.html, accessed on 1 April 2021) [19] when MES was not informative. Potential spliceogenic variants were selected according to the following criteria: (i) MES score changes $(>15 \%)[16,20]$ and (ii) creation of alternative sites (Table S2).

Four in silico approaches were used to predict variant-induced modifications in splicing regulatory elements: (a) HEXplorer $(\Delta \mathrm{HZ} \mathrm{EI}$; cut-off $<-5)$ (https: / / www2.hhu. de/rna/html/hexplorer_score.php, accessed on 1 April 2021) [21]; (b) Hot-Skip (https: / /hot-skip.img.cas.cz/, cut-off $\geq 1$, accessed on 1 April 2021) or Ex-Skip (https:/ / ex-skip. img.cas.cz/, accessed on 1 April 2021) [22]; (c) calculation of total ESRseq score changes ( $\Delta$ tESRseq) by quantitative assessment of RNA hexamers (cut-off < - 0.75) [23]; and (d) alteration of the ESE/ESS balance by HSF at Genomnis (https:/ /hsf.genomnis.com/home, accessed on 1 April 2021).

\subsection{Minigene Construction and Site-Directed Mutagenesis}

A 3591-bp fragment including RAD51D exons 2 to 9 and flanking introns was generated by DNA synthesis (Genewiz, South Plainfield, NJ, USA). This insert was cloned into the splicing plasmid pSAD [24-26] (minigene mgR51D_ex2-9, Figure 1, Figure S2). All sequence variations were introduced into mgR51D_ex2-9 by site-directed mutagenesis using the QuikChange Lightning kit (Agilent, Santa Clara, CA, USA) (Table S1). All constructs were confirmed by Sanger sequencing (Macrogen, Madrid, Spain).

\subsection{Functional Assays}

MCF-7 cell growth, transfection and inhibition of the nonsense-mediated decay were performed as previously described [16]. The triple-negative breast cancer (TNBC) cell line MDA-MB-231 was cultured in Dulbecco's Modified Eagle Medium (DMEM) supplemented with $10 \%$ fetal bovine serum, $2 \mathrm{mM}$ glutamine, $1 \%$ non-essential amino acids and $1 \%$ penicillin/streptomycin solution.

RNA was purified using the Genematrix Universal RNA Purification Kit (EURx, Gdansk, Poland), with on-column DNAse I digestion. RNA (400 ng) was retrotranscribed using the vector exon V2-specific primer RTPSPL3-RV (5'-TGAGGAGTGAATTGGTCGAA$3^{\prime}$ ) and the RevertAid First-Strand cDNA Synthesis Kit (Life Technologies, Carlsbad, CA, USA), following the manufacturer's instructions. Two $\mu \mathrm{L}$ of cDNA were used for amplification of the regions of interest using Platinum Taq polymerase (Life Technologies). To increase specificity, cDNA was amplified, with one primer located in one RAD51C exon of the insert and another one in a vector exon (V1 or V2). Thus, for variants of the donor site of exon 2, the amplification was performed using primers SD6-PSPL3_RTFW ( $5^{\prime}$ TCACCTGGACAACCTCAAAG-3') and RTR51D_9-rv (5'-GTCCCTGTCTCGAGTTATG-3') (amplicon size $=843 \mathrm{bp}$ ). For the remaining variants (exons 3 to 8 ), the following primers were used: RTR51D_ex2-fw (5'-TAGCTCAGAAATGTGGCTT-3') and RTpSAD-RV (Patent P201231427, CSIC) $($ size $=885 \mathrm{bp})$. Samples were denatured at $94{ }^{\circ} \mathrm{C}$ for $2 \mathrm{~min}$, followed 
by 35 cycles $\times\left(94{ }^{\circ} \mathrm{C}, 30 \mathrm{~s} / 59{ }^{\circ} \mathrm{C}, 30 \mathrm{~s} / 72{ }^{\circ} \mathrm{C}, 1 \mathrm{~min} / \mathrm{kb}\right)$ and $72{ }^{\circ} \mathrm{C}$ for $5 \mathrm{~min}$. RT-PCR products were sequenced by Macrogen (Madrid, Spain).

A)

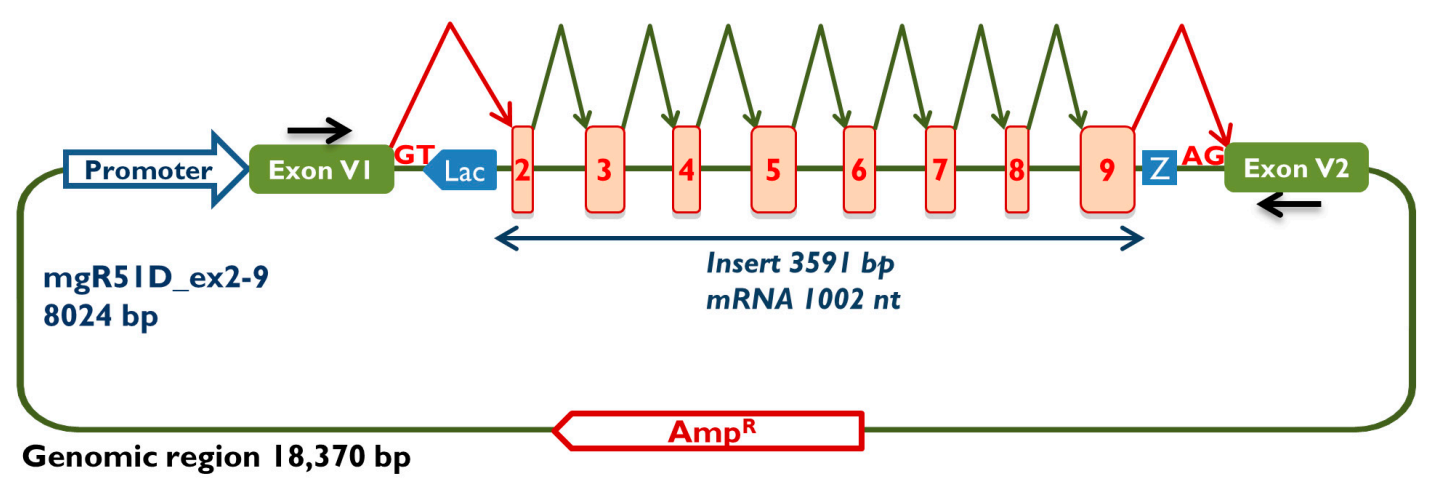

B)

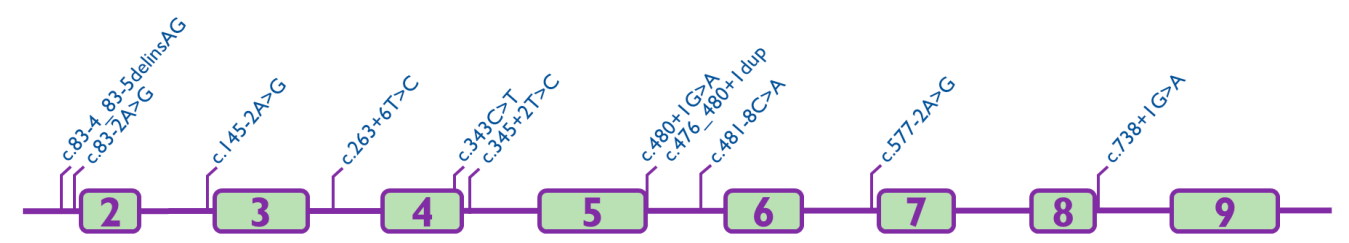

C)

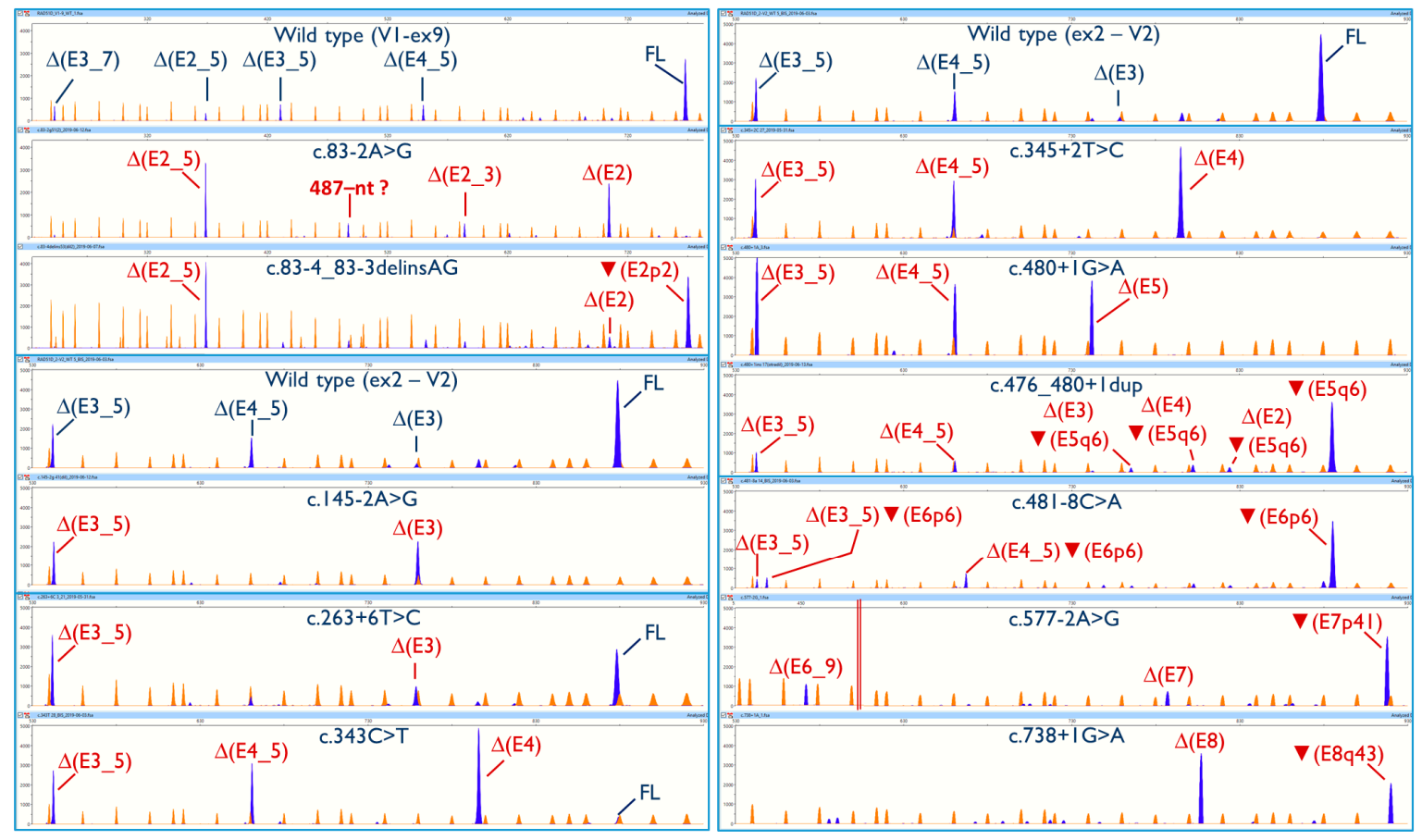

Figure 1. Structure of the minigene mgR51D_ex2-9 and functional assays of splice-site RAD51D variants. (A) Schematic representation of the RAD51D minigene, with exons 2 to 9. (B) Map of variants. (C) Fluorescent fragment analysis of transcripts generated by the wild type and mutant minigenes. FAM-labeled products (blue peaks) were run with LIZ-1200 (orange peaks) as size standard. FL: full-length transcript.

To estimate the relative proportions of each transcript, semi-quantitative fluorescent RT-PCRs were performed using a FAM-labeled primer under standard conditions, except that 26 cycles were herein applied $[14,27,28]$. Fluorescent products were run with LIZ-1200 
Size Standard by Macrogen (Seoul, Korea) and analyzed using Peak Scanner software V1.0 (Life Technologies). The mean peak areas of three independent experiments of each variant were used to calculate the relative proportions of each transcript as well as the standard deviations.

\subsection{ACMG/AMP-Like Classification of 37 RAD51D Variants Detected in BRIDGES Samples}

No RAD51D expert panel specifications of the American College of Medical Genetics and Genomics or the Association for Molecular Pathology (ACMG/AMP) variant curation guidelines were currently available (www.clinicalgenome.org, last accessed on 29 December 2020). Therefore, we performed a tentative classification based on: (i) generic ACMG/AMP guidelines [29], (ii) specific recommendations of the ClinGen Sequence Variant Interpretation Working Group for interpreting the loss-of-function PVS1 [30] and functional PS3/BS3 evidence codes [31], (iii) some non-gene-specific approaches proposed by the ClinGen CDH1 variant curation expert panel [32] and (iv) ad hoc rules based on expert judgment. The latter were essential to assign a specific functional splicing code (PS3/BS3) to complex minigene readouts (Supplementary Methods). In addition to PS3/BS3, only the rarity code (PM2) made a significant contribution to the classification process.

\section{Results}

\subsection{Bioinformatics Analysis and Functional Assays}

Forty-seven RAD51D variants detected in BRIDGES subjects were collected from the intron-exon boundaries of exons 2 to 9 and analyzed using MaxEntScan (MES) or NNSplice (Table S2). Eleven potential splice-site disrupting variants were selected to be assayed in MCF-7 cells (Table 1).

A RAD51D minigene with exons 2-9 was constructed in splicing vector pSAD (minigene mgR51D_ex2-9, Figures 1 and S2). Fragment analysis of the wild type (wt) minigene revealed the full-length (FL) transcript (V1-RAD51D exons 2 to 9-V2) and several alternative isoforms (Table 1; Figure 1C), of which $\Delta\left(\mathrm{E} 2 \_5\right), \Delta\left(\mathrm{E} 3 \_5\right)$ and $\Delta(\mathrm{E} 4$ _5), among others, were formerly reported as naturally occurring transcripts [33]. All 11 variants impaired splicing, wherein the FL transcript was (almost) absent in 10 variants and c. $263+6 \mathrm{~T}>\mathrm{C}$ still expressed a considerable level of FL transcript (49\%). Eight of these variants (c.263+6T >C, c.343C $>\mathrm{T}$, c.345+2T>C, c.480+1G>A, c.476_480+1dup, c.481-8C>A, c.577-2A $>$ G and c.738+1G>A) were also tested in the triple-negative breast cancer cells MDA-MB-231, in which they replicated the splicing profiles (Figure S3).

Five variants disrupted the canonical $3^{\prime}$ splice site ( $\left.3^{\prime} \mathrm{SS}\right)$, although c.83-4_83-3delinsAG and c.481-8C $>$ A also created a de novo site (transcripts (E2p2) and $\mathbf{\nabla}(\mathrm{E} 6 \mathrm{p} 6)$, respectively), and variant c.577-2A $>$ G mainly provoked the use of an intronic cryptic $3^{\prime} S S$ $(\boldsymbol{\nabla}(\mathrm{E} 7 \mathrm{p} 41))$. Four variants disrupted the canonical $5^{\prime}$ splice site $\left(5^{\prime} \mathrm{SS}\right): \mathrm{c} .345+2 \mathrm{~T}>\mathrm{C}$, c. $480+1 G>A$, c.476_480+1dup and c.738+1G>A. Variants c.476_480+1dup and c.738+1G>A also caused the recognition of alternative 5'SS ( $\mathbf{\nabla}(\mathrm{E} 5 \mathrm{q} 6)$ and $\mathbf{\nabla}(\mathrm{E} 8 \mathrm{q} 43)$, respectively). Finally, c.263+6T $>C$ and c.343C $>$ T weakened the natural 5'SS of exons 3 and 4, respectively.

\subsection{Transcript Analysis}

Our minigene analysis identified 25 transcripts, including the alternative ones generated by the wt minigene (Table S3 and Figure S4). Exon (or multi-exon) skipping was a frequent outcome, with 13 different aberrant transcripts detected: $\Delta(\mathrm{E} 2), \Delta\left(\mathrm{E} 2 \_3\right), \Delta\left(\mathrm{E} 2 \_5\right)$, $\Delta(\mathrm{E} 3), \Delta\left(\mathrm{E} 3 \_5\right), \Delta\left(\mathrm{E} 3 \_7\right), \Delta(\mathrm{E} 4), \Delta\left(\mathrm{E} 4 \_5\right), \Delta\left(\mathrm{E} 4 \_7\right), \Delta(\mathrm{E} 5), \Delta\left(\mathrm{E} 6 \_9\right), \Delta(\mathrm{E} 7)$ and $\Delta(\mathrm{E} 8)$. Another five aberrant transcripts were due to the use of de novo splice sites ( $\mathbf{\nabla}(\mathrm{E} 2 \mathrm{p} 2), \boldsymbol{\nabla}(\mathrm{E} 5 \mathrm{q} 6)$, $\boldsymbol{\nabla}(\mathrm{E} 6 \mathrm{p} 6), \boldsymbol{\nabla}(\mathrm{E} 7 \mathrm{p} 41)$ and $\boldsymbol{\nabla}(\mathrm{E} 8 \mathrm{q} 43))$, and five aberrant transcripts combined skipping and alternative site usage events $\left((\Delta(\mathrm{E} 2) \boldsymbol{\nabla}(\mathrm{E} 5 \mathrm{q} 6)),(\Delta(\mathrm{E} 3) \boldsymbol{\nabla}(\mathrm{E} 5 \mathrm{q} 6)),\left(\Delta\left(\mathrm{E} 3 \_5\right) \boldsymbol{\nabla}(\mathrm{E} 6 \mathrm{p} 6)\right),(\Delta(\mathrm{E} 4)\right.$ $\boldsymbol{\nabla}(\mathrm{E} 5 \mathrm{q} 6))$ and $\left(\Delta\left(\mathrm{E} 4 \_5\right) \boldsymbol{\nabla}(\mathrm{E} 6 \mathrm{p} 6)\right)$. Two additional fragments of 487- and 1363-nt were also found, but the presumed aberrant events could not be characterized. Seventeen transcripts introduced PTCs that were predicted to inactivate RAD51D, and six kept the reading frame (Table 1, Supplementary Methods). 
Table 1. Bioinformatics analysis and splicing outcomes of $R A D 51 D$ canonical splice variants.

\begin{tabular}{|c|c|c|c|c|c|}
\hline \multirow{2}{*}{ Variant (HGVS) $^{1}$} & \multirow{2}{*}{ Bioinformatics (MaxEnt Scan) ${ }^{2}$} & \multicolumn{4}{|c|}{ Transcripts $^{3}$} \\
\hline & & Canonical & PTC & In-Frame & Uncharacterized \\
\hline mgR51D_ex2-9 & Primers V1-ex9 & & & & \\
\hline Wild type & - & $57.8 \% \pm 5.6$ & $\begin{array}{l}\Delta(\text { E4_5) }(13.8 \% \pm 1.3) \\
\Delta(\text { E3_7) }(9.9 \% \pm 2.6) \\
\Delta(\text { E2_5) }(5.2 \% \pm 0.8)\end{array}$ & $\Delta$ (E3_5) $(13.3 \% \pm 2.4)$ & - \\
\hline c. $83-2 A>G$ & {$[-] 3^{\prime} \mathrm{SS}(8.52 \rightarrow 0.56)$} & - & $\begin{array}{c}\Delta(\mathrm{E} 2-5)(41.9 \% \pm 1.1) \\
\Delta(\mathrm{E} 2)(39.6 \% \pm 0.9) ; \\
\Delta\left(\mathrm{E} 2 \_3\right)(9.6 \% \pm 0.1)\end{array}$ & - & 487-nt $(9.0 \% \pm 0.2)$ \\
\hline c.83-4_83-3delinsAG & $\begin{array}{c}{[-] 3^{\prime} \mathrm{SS}} \\
{[+] 3^{\prime} \mathrm{SS}(5.42) 2 \text {-nt upstream }}\end{array}$ & - & $\begin{array}{l}\mathbf{\nabla}(\mathrm{E} 2 \mathrm{p} 2)(54.2 \% \pm 2.0) \\
\Delta(\mathrm{E} 25)(38.6 \% \pm 2.1) \\
\Delta(\mathrm{E} 2)(7.2 \% \pm 0.4)\end{array}$ & - & - \\
\hline mgR51D_ex2-9 & Primers ex2-V2 & & & - & - \\
\hline Wild type & & $73.1 \% \pm 5.6$ & $\Delta\left(\mathrm{E} 4 \_5\right)(9.4 \% \pm 3)$ & $\Delta\left(\mathrm{E} 3 \_5\right)(17.5 \% \pm 5.2)$ & - \\
\hline c.145-2A>G & {$[-] 3^{\prime} S S(2.43)$} & - & $\Delta(\mathrm{E} 3)(55.5 \% \pm 0.6)$ & $\Delta\left(\mathrm{E} 3 \_5\right)(44.5 \% \pm 0.6)$ & - \\
\hline$c .263+6 \mathrm{~T}>\mathrm{C}$ & 5'SS: $7.44 \rightarrow 4.86$ & $49.0 \% \pm 1.2$ & $\Delta(\mathrm{E} 3)(15.6 \% \pm 0.3)$ & $\Delta\left(\mathrm{E} 3 \_5\right)(35.4 \% \pm 1.2)$ & - \\
\hline c. $343 \mathrm{C}>\mathrm{T}$ & 5'SS: $7.79 \rightarrow 4.36$ & $3.4 \% \pm 0.7$ & $\begin{array}{c}\Delta(\mathrm{E} 4)(45.7 \% \pm 1.0) \\
\Delta\left(\mathrm{E} 4 \_5\right)(28.1 \% \pm 0.6)\end{array}$ & $\Delta\left(\mathrm{E} 3 \_5\right)(22.7 \% \pm 1.2)$ & - \\
\hline c. $345+2 \mathrm{~T}>\mathrm{C}$ & {$[-] 5^{\prime}$ SS $(7.79 \rightarrow 0.04)$} & - & $\begin{array}{c}\Delta(\mathrm{E} 4)(49.6 \% \pm 1.4) \\
\Delta\left(\mathrm{E} 4 \_5\right)(26.4 \% \pm 1.0)\end{array}$ & $\Delta\left(\mathrm{E} 3 \_5\right)(24.0 \% \pm 0.7)$ & - \\
\hline c.476_480+1dup & {$[-] 5^{\prime} S S(11.08 \rightarrow 0.5)$} & - & $\begin{array}{c}\Delta(\mathrm{E} 45)(8.6 \% \pm 0.4) \\
(\Delta(\mathrm{E} 4) \mathbf{\nabla}(\mathrm{E} 5 \mathrm{q} 6))(6.3 \% \pm 0.1) \\
(\Delta(\mathrm{E} 2) \mathbf{\nabla}(\mathrm{E} 5 \mathrm{q} 6))(4.3 \% \pm 0.1) \\
(\Delta(\mathrm{E} 3) \mathbf{\nabla}(\mathrm{E} 5 \mathrm{q} 6))(3.6 \% \pm 0.4)\end{array}$ & $\begin{array}{l}\mathbf{\nabla}(\mathrm{E} 5 \mathrm{q} 6)(60.4 \% \pm 4.3) \\
\Delta\left(\mathrm{E} 3 \_5\right)(16.7 \% \pm 5.0)\end{array}$ & - \\
\hline c. $481-8 \mathrm{C}>\mathrm{A}$ & $\begin{array}{l}\text { 3'SS: } 8.21 \rightarrow 1.75 \\
{[+] \text { 3'SS (11.06) 6-nt upstream }}\end{array}$ & $0.4 \% \pm 0.1$ & $\left(\Delta\left(\mathrm{E} 4 \_5\right) \mathbf{\nabla}(\mathrm{E} 6 \mathrm{p} 6)\right)(13.4 \% \pm 0.2)$ & $\begin{array}{c}\mathbf{\nabla}(\text { E6p6) }(70.0 \% \pm 0.8) ;(\Delta(\text { E3_5 }) \\
\mathbf{\nabla}(\text { E6p6) })(8.8 \% \pm 0.3) \\
\Delta(\text { E3_5) }(7.5 \% \pm 0.3)\end{array}$ & - \\
\hline c. $577-2 \mathrm{~A}>\mathrm{G}$ & {$[-] 3^{\prime}$ SS $(10.36 \rightarrow 2.41)$} & - & $\begin{array}{l}\mathbf{\nabla}(\text { E7p41) }(65.5 \% \pm 0.5) \\
\Delta(\text { E7) }(14.3 \% \pm 0.2)\end{array}$ & $\Delta\left(\mathrm{E} 6 \_9\right)(20.2 \% \pm 0.8)$ & - \\
\hline c. $738+1 G>A^{4}$ & {$[-] 5^{\prime}$ SS $(6.13 \rightarrow-2.05)$} & - & $\begin{array}{c}\mathbf{\nabla}(\mathrm{E} 8 \mathrm{q} 43)(27.9 \% \pm 0.1) \\
\Delta(\mathrm{E} 8)(51.1 \% \pm 0.2) \\
\Delta\left(\mathrm{E} 4 \_7\right)(10.9 \% \pm 0.1)\end{array}$ & - & $1363-$ nt $(10.1 \% \pm 0.0)$ \\
\hline
\end{tabular}

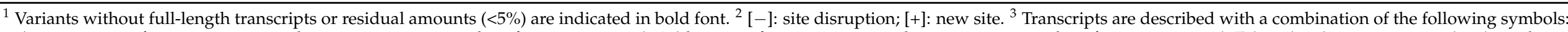

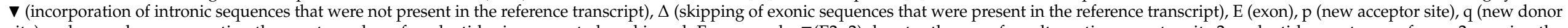

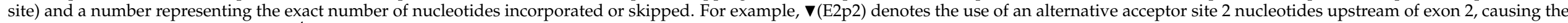

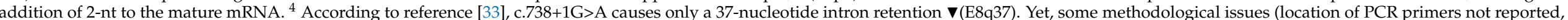


with our minigene results. 


\subsection{ESE Mapping}

Effective exon recognition in alternative splicing requires the binding of trans-acting factors to exon-splicing enhancer (ESE) sequences [34]. Exon 3 is a non-constitutive exon, excluded from several $R A D 51 D$ naturally occurring, alternatively spliced isoforms [33] and consequently, it is likely regulated by splicing regulatory elements (SRE) [35]. We proceeded to functionally map SREs in exon 3 by introducing four overlapping 30-35-bp deletions (c.147_176del, c.172_206del, c.202_235del and c.231_260del) along this exon (119 $\mathrm{bp}$ ), excluding the first two and last three nucleotides to preserve splice site-conserved positions [24,25]. All four deletions had an impact on splicing, and different levels of exon 3 skipping were observed (Figure 2A,B; Table S4; Figure S5), pointing out that these sequences might have contained regulatory motifs. Deletion c.202_235del affected splicing the most, observing no trace of the FL transcript (Figure 2A,B).

We then decided to functionally assay 25 BRIDGES variants reported at the most spliceogenic segment, c.202_235del, and surrounding sequences. In this study, variants with a FL transcript proportion ranging from 65.8 to $73.1 \%(<10 \%$ reduction of the FL transcript in the wt minigene) were not considered spliceogenic (see Section 4 ). In total, 16 out of 25 variants (64\%) effectively disrupted splicing (Table 2; Figures 2A,B and S5), wherein exon 3 skipping (not observed in the wt minigene) was detected and $\Delta$ (E3_5) was increased $(>17.5 \%)$ in all the spliceogenic variants. In addition, c.202G $>$ A also expressed a new isoform, $\Delta$ (E3p36), through the use of an internal cryptic acceptor site. Remarkably, c. $202 \mathrm{G}>\mathrm{A}$ was the only variant that did not produce FL transcripts, whereas c.214T>C produced $100 \%$ full-length transcripts.

To refine the SRE map and identify potential hotspots for spliceogenic variants, five additional overlapping 10/11-bp deletions of the c.202_235 interval were tested. All deletions impaired splicing, being particularly significant in c.210_219del, wherein the FL transcript was absent (Table S4, Figure S5). Next, we tested all the possible changes of the highest spliceogenic interval, c.210_219. We selected the six specific nucleotides, i.e., c.212-217, within this segment that were not shared with the overlapping microdeletions c.202_211del and c.218_227del (see Figure 2A). BRIDGES variants c.213C $>$ T, c.214T >C, c. $216 \mathrm{C}>\mathrm{T}$ and c.217G $>\mathrm{A}$ of this segment had been already tested in the preliminary SRE assays (Table 2). Next, the 14 remaining possible nucleotide substitutions in segment c.212c. 217 were introduced into the minigene and evaluated. They represented the so-called "artificial" (ad hoc) variants that had not been identified in BRIDGES subjects. Eleven artificial variants within this interval impaired splicing, reducing the FL transcript by $\geq 10 \%$, inducing the aberrant transcript $\Delta$ (E3) and increasing the relative proportion of the alternative isoform $\Delta$ (E3_5). Putting together the artificial and BRIDGES variants of c. $212-217$, the final proportion of spliceogenic variants was $66.7 \%(12 / 18)$.

In order to assess the accuracy of four ESE/ESS prediction tools (Human Splicing Finder (HSF), HEXplorer, Hot-Skip and $\triangle$ tESRseq), we pooled together and analyzed the 25 BRIDGES variants and the 14 artificial ones of the c.212-217 segment (Table 2; Tables S5 and S6) [36,37]. HEXplorer showed the highest sensitivity $(74.1 \%, 20 / 27)$ and accuracy $(61.5 \%)$ but also showed the lowest specificity (33.3\%). The sensitivity of the other three predictors was very low $(<37 \%)$. On the other hand, Hot-Skip presented the highest specificity (83.3\%), but also 19 false negative variants. Remarkably, the spliceogenic variant c.199A $>$ G would not have been predicted by any tool. Moreover, Hot-Skip analysis of the full exon 3 sequence suggested three changes with the highest probabilities of splicing disruption: c.163C $>$ T (detected in BRIDGES subjects), c.163C $>\mathrm{G}$ and c.178C $>$ T. We tested them in our RAD51D minigene, where they had weak-to-moderate effects, generating different quantities of the FL isoform (38.7-62.0\%; Table 2).

Summarizing, we evaluated a total of 42 candidate ESE variants (26 identified in BRIDGES subjects), 30 of which impaired RAD51D exon 3 splicing. 


\subsection{ACMG/AMP-Like Classification of 37 RAD51D Variants Identified in the BRIDGES Cohort}

We developed ACMG/AMP-based specifications for the interpretation of RAD51D spliceogenic variants (see Supplementary Methods, Figures S6-S8). With this approach, based on minigene read-outs (PS3/BS3 codes) and gnomADv2.1 global population frequencies (PM2/BS1/BA1 codes), we classified 37 BRIDGES variants (Table 3). As expected, most variants located at canonical splice sites (9 of 11) ended up as likely pathogenic. By contrast, many of the remaining variants ended up as variants of uncertain significance (VUS), despite demonstrating an effect on splicing.

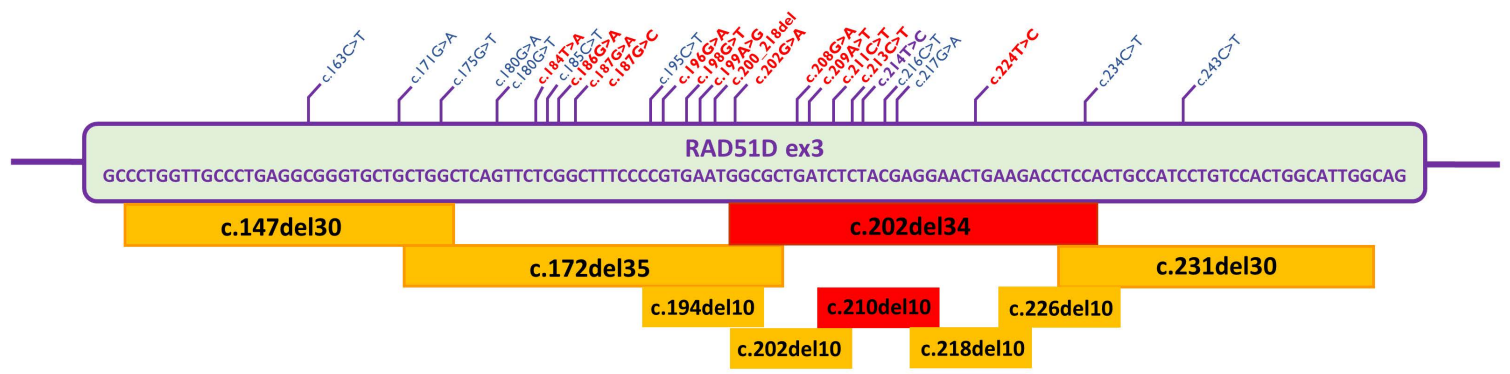

B)


Figure 2. Analysis of RAD51D exon 3 variants. (A) Map of exon 3 microdeletions and tested variants. Boxes: red, total splicing disruptions; yellow, weak to moderate disruptions. Spliceogenic variants are shown in red. (B) Fluorescent fragment analysis of transcripts generated by selected microdeletions (left) and variants (right). FAM-labeled products (blue peaks) were run with LIZ-1200 (orange peaks) as the size standard. 
Table 2. Bioinformatics analysis and splicing outcomes of putative exon 3 ESE variants.




Table 2. Cont.

\begin{tabular}{|c|c|c|c|c|c|c|c|}
\hline \multirow{2}{*}{ Variant $^{1}$} & \multicolumn{3}{|c|}{ Transcripts ${ }^{2}$} & \multicolumn{4}{|c|}{ In Silico Tools ${ }^{3}$} \\
\hline & Canonical & PTC & In-Frame & HSF & HEXplorer & Hot-Skip & $\Delta$ tESRseq \\
\hline c.224T $>C$ & $59.9 \% \pm 0.9$ & $\Delta\left(\mathrm{E} 4 \_5\right)(13.4 \% \pm 0.1)$ & $\Delta$ (E3_5) $(26.7 \% \pm 0.8)$ & - & -9.3 & 0 & 0.27 \\
\hline $\mathrm{c} .234 \mathrm{C}>\mathrm{T}$ & $70.7 \% \pm 0.5$ & $\Delta\left(\mathrm{E} 4 \_5\right)(11.5 \% \pm 0.2)$ & $\Delta\left(\mathrm{E} 3 \_5\right)(17.8 \% \pm 0.4)$ & + & -0.3 & 0 & 0.10 \\
\hline c. $243 \mathrm{C}>\mathrm{T}$ & $50.9 \% \pm 1.2$ & $\Delta$ (E3) $(16.8 \% \pm 0.1)$ & $\Delta\left(E 3 \_5\right)(32.3 \% \pm 1.1)$ & - & -59.7 & 4 & -0.58 \\
\hline \multicolumn{8}{|c|}{ Artificial Variants c.212_217 } \\
\hline c.212T $>\mathrm{A}$ & $63.6 \% \pm 2.3$ & $\begin{array}{c}\Delta(\mathrm{E} 3)(5.5 \% \pm 0.3) \\
\Delta\left(\mathrm{E} 4 \_5\right)(5.5 \% \pm 0.0)\end{array}$ & $\Delta\left(E 3 \_5\right)(25.4 \% \pm 2.6)$ & + & 16.1 & 0 & 0.51 \\
\hline c.212T $>C$ & $62.7 \% \pm 1.0$ & $\begin{array}{c}\Delta(\mathrm{E} 3)(5.5 \% \pm 0.1) \\
\Delta\left(\mathrm{E} 4 \_5\right)(7.1 \% \pm 0.0)\end{array}$ & $\Delta\left(E 3 \_5\right)(24.7 \% \pm 0.9)$ & + & -0.4 & 0 & -0.01 \\
\hline$c .212 \mathrm{~T}>\mathrm{G}$ & $63.4 \% \pm 2.1$ & $\begin{array}{c}\Delta(\mathrm{E} 3)(5.9 \% \pm 0.2) \\
\Delta\left(\mathrm{E} 4 \_5\right)(4.9 \% \pm 0.2)\end{array}$ & $\Delta\left(E 3 \_5\right)(25.8 \% \pm 1.9)$ & + & 41.8 & 0 & 1.58 \\
\hline c. $213 \mathrm{C}>\mathrm{A}$ & $41.4 \% \pm 0.3$ & $\begin{array}{c}\Delta(\mathrm{E} 3)(11.1 \% \pm 0.2) \\
\Delta\left(\mathrm{E} 4 \_5\right)(6.4 \% \pm 0.0)\end{array}$ & $\Delta\left(E 3 \_5\right)(41.1 \% \pm 0.5)$ & - & -25.6 & 0.5 & -0.81 \\
\hline c. $213 C>G$ & $49.6 \% \pm 0.6$ & $\begin{array}{c}\Delta(\text { E3) }(6.7 \% \pm 0.1) \\
\Delta(\text { E4_5) }(6.1 \% \pm 0.0)\end{array}$ & $\Delta\left(E 3 \_5\right)(37.6 \% \pm 0.7)$ & - & -20.1 & 0.5 & 0.57 \\
\hline c. $214 \mathrm{~T}>\mathrm{A}$ & $64.3 \% \pm 1.3$ & $\Delta\left(\mathrm{E} 4 \_5\right)(10.9 \% \pm 0.1)$ & $\Delta\left(E 3 \_5\right)(24.8 \% \pm 1.3)$ & + & 19.1 & 0 & 0.87 \\
\hline c. $215 \mathrm{~A}>\mathrm{C}$ & $28.7 \% \pm 0.2$ & $\Delta\left(\mathrm{E} 4 \_5\right)(9.6 \% \pm 0.3)$ & $\Delta$ (E3_5) $(61.7 \% \pm 0.4)$ & - & -40.5 & 0 & 1.82 \\
\hline c. $215 A>G$ & $69.9 \% \pm 1.6$ & $\Delta\left(\mathrm{E} 4 \_5\right)(9.4 \% \pm 0.2)$ & $\Delta\left(E 3 \_5\right)(20.7 \% \pm 1.5)$ & - & -27.9 & 0.33 & 1.78 \\
\hline c. $215 \mathrm{~A}>\mathrm{T}$ & $70.6 \% \pm 1.7$ & $\Delta\left(\mathrm{E} 4 \_5\right)(8.7 \% \pm 0.1)$ & $\Delta\left(\mathrm{E} 3 \_5\right)(20.7 \% \pm 1.6)$ & - & -21.2 & 0.67 & 1.90 \\
\hline c. $216 \mathrm{C}>\mathrm{A}$ & $54.7 \% \pm 1.0$ & $\Delta$ (E3) $(12.8 \% \pm 0.4)$ & $\Delta\left(E 3 \_5\right)(32.5 \% \pm 1.4)$ & - & -73.7 & 0.13 & -0.56 \\
\hline c. $216 C>G$ & $36.4 \% \pm 0.6$ & $\Delta$ (E3) $(22.1 \% \pm 1.3)$ & $\Delta\left(\mathbf{E 3} \_5\right)(41.6 \% \pm 1.9)$ & - & -91 & 0.33 & -1.15 \\
\hline c. $217 G>C$ & $62.7 \% \pm 0.5$ & $\Delta$ (E3) $(8.3 \% \pm 0.1)$ & $\Delta$ (E3_5) $(29.0 \% \pm 0.4)$ & - & -21.2 & 0 & -0.27 \\
\hline c. $217 \mathrm{G}>\mathrm{T}$ & $63.6 \% \pm 0.4$ & $\Delta$ (E3) $(14.3 \% \pm 0.3)$ & $\Delta\left(\mathrm{E} 3 \_5\right)(22.1 \% \pm 0.5)$ & - & -114.2 & 1.67 & -1.66 \\
\hline \multicolumn{8}{|c|}{ Hot-Skip Variants } \\
\hline c. $163 C>G$ & $38.7 \% \pm 3.0$ & $\Delta$ (E3) $(26.9 \% \pm 0.7)$ & $\Delta($ E3_5) $(34.4 \% \pm 2.5)$ & - & -92.7 & 18 & -2.81 \\
\hline $\begin{array}{c}\text { c.163C>T } \\
\text { (BRIDGES) }\end{array}$ & $62.0 \% \pm 0.5$ & $\begin{array}{c}\Delta\left(\mathrm{E} 4 \_5\right)(12.3 \% \pm 0.3) \\
\Delta(\mathrm{E} 3)(5.6 \% \pm 0.1)\end{array}$ & $\Delta\left(\mathrm{E} 3 \_5\right)(20.1 \% \pm 0.6)$ & $\begin{array}{c}- \\
\text { MES: new 5'SS } \\
(7.07)\end{array}$ & -52.0 & 16 & -1.978 \\
\hline
\end{tabular}


Table 2. Cont.

\begin{tabular}{|c|c|c|c|c|c|c|c|}
\hline \multirow{2}{*}{ Variant $^{1}$} & \multicolumn{3}{|c|}{ Transcripts ${ }^{2}$} & \multicolumn{4}{|c|}{ In Silico Tools ${ }^{3}$} \\
\hline & Canonical & PTC & In-Frame & HSF & HEXplorer & Hot-Skip & $\Delta$ tESRseq \\
\hline c. $178 \mathrm{C}>\mathrm{T}$ & $54.3 \% \pm 1.0$ & $\begin{array}{c}\Delta \text { (E3) }(16.0 \% \pm 0.1) \\
\Delta(\text { E4_5) }(4.8 \% \pm 0.1)\end{array}$ & $\Delta($ E3_5) $(24.9 \% \pm 0.8)$ & - & -44.7 & 12 & -2.08 \\
\hline
\end{tabular}

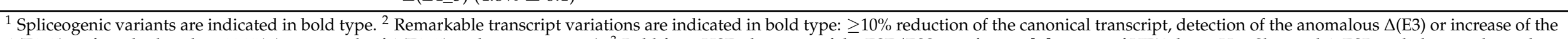

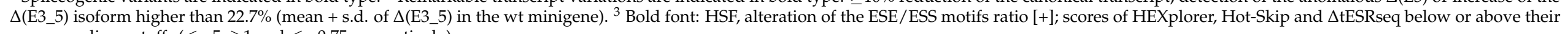
corresponding cutoffs $(\leq-5, \geq 1$ and $\leq-0.75$, respectively).

Table 3. Proposed pSAD-based ACMG/AMP clinical classification of 37 RAD51D genetic variants detected in the BRIDGES cohort.

\begin{tabular}{|c|c|c|c|c|c|c|}
\hline c.HGVS ${ }^{1}$ & p.HGVS ${ }^{1}$ & Clinvar ${ }^{2}$ & PS3/BS3 ${ }^{3}$ & PM2/BS1/BA1 4 & Proxy for Allele Counts ${ }^{5}$ & Variant Classification $^{6}$ \\
\hline c.83-4_-3delinsAG & p.? & VUS $(* *)$ & PS3_VS & (0/251433) PM2 & rs780590372 (=) & Likely Pathogenic (PS3_VS + PM2) \\
\hline c. $83-2 A>G$ & p.? & $\mathrm{LP}(*)$ & PS3_VS $\left(91 \% \mathrm{PS} 3\right.$ VS + 9\%N/A) ${ }^{7}$ & (0/251433) PM2 & rs780590372 (-1) & Likely Pathogenic (PS3_VS + PM2) \\
\hline c. $145-2 A>G$ & p.? & not reported & PS3_VS & (0/251102) PM2 & rs201974522 (-1) & Likely Pathogenic (PS3_VS + PM2) \\
\hline c. $163 \mathrm{C}>\mathrm{T}$ & p.(Arg55Trp) & VUS $(* *)$ & N/A (62\%N/A + 38\%PS3_VS) & (2/251433) PM2 & - & Uncertain Significance (PM2 only) \\
\hline c. $171 \mathrm{G}>\mathrm{A}$ & p.(Leu57=) & $\operatorname{LB}(* *)$ & BS3 (76\%BS3 + 24\%PS3_VS) & (0/249480) PM2 & rs745307359 (-4) & Uncertain Significance (BS3 + PM2) \\
\hline c. $175 \mathrm{G}>\mathrm{T}$ & p.(Ala59Ser) & not reported & N/A (53\%N/A + 47\%PS._VS) & (0/251298) PM2 & rs780689600 (+4) & Uncertain Significance (PM2 only) \\
\hline c. $180 \mathrm{G}>\mathrm{T}$ & p.(Gln60His) & VUS $(* *)$ & N/A (69\%N/A + 31\%PS3_VS) & (0/251298) PM2 & rs780689600 $(-1)$ & Uncertain Significance (PM2 only) \\
\hline c. $184 \mathrm{~T}>\mathrm{A}$ & p.(Ser62Thr) & not reported & N/A (37\%N/A + 63\%PS3_VS) & (0/251382) PM2 & rs374357106 (+3) & Uncertain Significance (PM2 only) \\
\hline c. $185 \mathrm{C}>\mathrm{T}$ & p.(Ser62Leu) & VUS $(* *)$ & N/A (66\%N/A + 34\%PS3_VS) & $(5 / 251382) \mathrm{N} / \mathrm{A}$ & - & Uncertain Significance (no codes) 8 \\
\hline c. $186 \mathrm{G}>\mathrm{A}$ & p.(Ser62=) & $\mathrm{LB}(* *)$ & N/A (64\%BS3 + 36\%PS3_VS) & $(3 / 251380) \mathrm{N} / \mathrm{A}$ & - & Uncertain Significance (no codes) 8 \\
\hline c. $187 \mathrm{G}>\mathrm{A}$ & p.(Ala63Thr) & VUS $(* *)$ & N/A (60\%N/A + 40\%PS3_VS) & $(1 / 251408)$ PM2 & - & Uncertain Significance (PM2 only) \\
\hline c. $187 \mathrm{G}>\mathrm{C}$ & p.(Ala63Pro) & not reported & N/A (27\%N/A + 73\%PS3_VS) & (0/251408) PM2 & c. $187 \mathrm{G}>\mathrm{A}$ & Uncertain Significance (PM2 only) \\
\hline c. $196 \mathrm{G}>\mathrm{A}$ & p.(Val66Met) & B (2), LB (4), VUS (4) & N/A (59\%N/A + 41\%PS3_VS) & $(80 / 251384)$ BS1 & - & Uncertain Significance (BS1) ${ }^{8}$ \\
\hline c. $198 \mathrm{G}>\mathrm{T}$ & p. $($ Val66=) & $\operatorname{LB}(* *)$ & N/A (49\%BS3 + 51\%PS3_VS) & $(9 / 251448) \mathrm{N} / \mathrm{A}$ & - & Uncertain Significance (no codes) 8 \\
\hline c. $199 \mathrm{~A}>\mathrm{G}$ & p.(Asn67Asp) & not reported & N/A (65\%N/A + 35\%PS3_VS) & (0/251448) PM2 & rs546461804 (+1) & Uncertain Significance (PM2 only) \\
\hline c.200_218del & p.(Asn67Argfs*7) & not reported & PS3_VS & (0/251448) PM2 & rs54661804 (=) & Likely Pathogenic (PS3_VS + PM2) \\
\hline
\end{tabular}


Table 3. Cont.

\begin{tabular}{|c|c|c|c|c|c|c|}
\hline c.HGVS ${ }^{1}$ & p.HGVS ${ }^{1}$ & Clinvar $^{2}$ & $\mathrm{PS} / \mathrm{BS}^{3}{ }^{3}$ & PM2/BS1/BA1 ${ }^{4}$ & Proxy for Allele Counts ${ }^{5}$ & Variant Classification $^{6}$ \\
\hline c. $202 \mathrm{G}>\mathrm{A}$ & p.(Gly68Ser) & VUS $(* *)$ & PS3 (68\%PS3_VS + 32\%PS3) & $(9 / 251454) \mathrm{N} / \mathrm{A}$ & - & Uncertain Significance (PS3 only) \\
\hline c.208G >A & p.(Asp70Asn) & VUS $(* *)$ & N/A (47\%N/A + 53\%PS3_VS) & $(8 / 251458) \mathrm{N} / \mathrm{A}$ & - & Uncertain Significance (no codes) ${ }^{8}$ \\
\hline c. $209 \mathrm{~A}>\mathrm{T}$ & p.(Asp70Val) & VUS $(* *)$ & N/A (58\%N/A + 42\%PS3_VS) & $(1 / 251450)$ PM2 & - & Uncertain Significance (PM2 only) $^{8}$ \\
\hline c. $211 \mathrm{C}>\mathrm{T}$ & p.(Leu71Phe) & VUS $\left(^{*}\right)$ & N/A (61\%N/A + 39\%PS3_VS) & $(0 / 251476)$ PM2 & rs559850711 (+1) & Uncertain Significance (PM2 only) ${ }^{8}$ \\
\hline c. $213 C>T$ & p. $($ Leu71=) & $\mathrm{LB}(* *)$ & N/A (57\%BS3 + 43\%PS3_VS) & $(2 / 251466)$ PM2 & - & Uncertain Significance (PM2 only) ${ }^{8}$ \\
\hline c. $214 \mathrm{~T}>\mathrm{C}$ & p.(Tyr72His) & not reported & $\mathrm{N} / \mathrm{A}$ & $(0 / 251466)$ PM2 & rs745546403 (-1) & Uncertain Significance (PM2 only) \\
\hline c. $217 \mathrm{G}>\mathrm{A}$ & p.(Glu73Lys) & VUS $(* *)$ & N/A (67\%N/A + 33\%PS3_VS) & $(2 / 251462)$ PM2 & - & Uncertain Significance (PM2 only) ${ }^{8}$ \\
\hline c. $224 \mathrm{~T}>\mathrm{C}$ & p.(Leu75Pro) & not reported & N/A (60\%N/A + 40\%PS3_VS) & (0/251466) PM2 & rs746929682 (-1) & Uncertain Significance (PM2 only) ${ }^{8}$ \\
\hline c. $234 \mathrm{C}>\mathrm{T}$ & p. $($ Ser78=) & $\mathrm{B}(* *)$ & BS3 (71\%BS3 + 29\%PS3_VS) & $(29273 / 251448)$ BA1 & - & Benign (BS3 + BA1) \\
\hline c. $243 C>T$ & p.(Ile81=) & $\mathrm{LB}(* *)$ & N/A (60\%BS3 + 40\%PS3_VS) & $(1 / 251472)$ PM2 & - & Uncertain Significance (PM2 only) ${ }^{8}$ \\
\hline c. $263+6 \mathrm{~T}>\mathrm{C}$ & p.? & not reported & N/A (49\%BS3 + 51\%PS3_VS) & $(0 / 251424)$ PM2 & rs56218020 (+1) & Uncertain Significance (PM2) ${ }^{8}$ \\
\hline c. $343 \mathrm{C}>\mathrm{T}$ & p.(Gln115Ter) & $\mathrm{P}(* *)$ & PS3_VS & (0/141295) PM2 & $\begin{array}{c}\text { rs786202507 } \\
(-4) r s 878854562(+7)\end{array}$ & Likely Pathogenic (PS3_VS + PM2) \\
\hline c.476_480+1dup & p.? & not reported & N/A (40\%PS3_VS + 60\%N/A) & $(0 / 251474)$ PM2 & rs1057521922 (=) & Uncertain Significance (PM2 only) ${ }^{8}$ \\
\hline c. $480+1 G>A$ & p.? & $(-)$ & PS3 (70\%PS3_VS + 30\%PS3) & $(0 / 251474)$ PM2 & rs1057521922 (-3) & Likely Pathogenic (PS3 + PM2) \\
\hline c. $481-8 \mathrm{C}>\mathrm{A}$ & p.? & not reported & N/A (30\%PS3_VS + 70\%N/A) & (0/241990) PM2 & rs762247126 (=) & Uncertain Significance (PM2 only) ${ }^{8}$ \\
\hline c. $577-2 \mathrm{~A}>\mathrm{G}$ & p.? & $\mathrm{P} / \mathrm{LP}(* *)$ & PS3 (80\%PS3_VS + 20\%PS3) & $(0 / 250980)$ PM2 & rs1210749655 (-4) & Likely Pathogenic (PS3 + PM2) \\
\hline c. $738+1 \mathrm{G}>\mathrm{A}$ & p.? & $\operatorname{LP}(* *)$ & $\begin{array}{c}\text { PS3_VS (70\%PS3_VS + } \\
10 \% \mathrm{~N} / \mathrm{A})^{7}\end{array}$ & (0/240992) PM2 & rs1210620444 (-1) & Likely Pathogenic (PS3 + PM2) ${ }^{9}$ \\
\hline
\end{tabular}

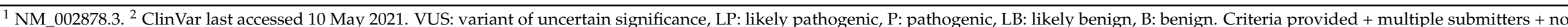

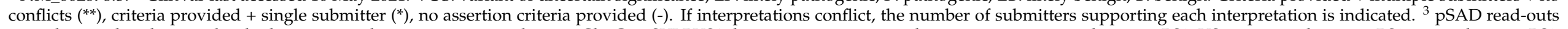



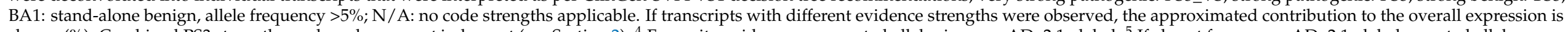

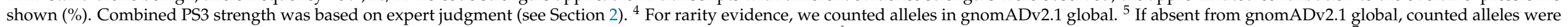

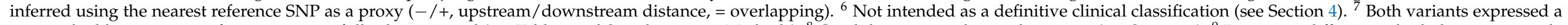

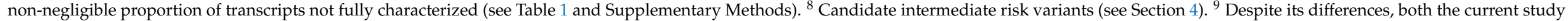
and references [38] support a likely pathogenic classification for this variant. 


\section{Discussion}

The progress in DNA sequencing technology has resulted in the development of multi-gene panels for clinical genetic testing of diverse genetic diseases, including hereditary breast cancer. Although next-generation sequencing is a powerful tool to identify potentially deleterious variants, most variants are classified as VUS because there are no existing datasets correlating them with their impact on gene function and cancer risk [39]. The goal of the BRIDGES initiative was to establish reliable breast cancer risk estimates for 34 putative breast cancer susceptibility genes commonly included in multigene panels by sequencing more than 60,000 breast cancer cases and 53,000 controls [5]. As a partner in this project, we have carried out the most comprehensive functional evaluation of candidate splice variants of RAD51D performed so far.

Despite the fact that assays using patient-derived RNA are considered the most suitable strategy to evaluate splicing outcomes, minigene assays have proved to be a useful, simple and robust approach to assess potential spliceogenic variants [12,40]. This method presents several significant advantages such as (1) analysis of single allele events without the meddling of the WT allele; (2) accurate quantification of isoforms by inhibiting the nonsense-mediated decay; (3) high versatility, allowing the study of different variants with a single minigene; (4) high reproducibility of physiological and pathological splicing patterns; (5) analysis in a cell type relevant for the disease.

In relation to the latter, we have performed all analyses in breast cancer cell line MCF-7, and we have performed a sub-analysis in the TN breast cancer cell line MDAMB-231, replicating findings (Figure S3). Current data supports a shared heritability for ER-negative (or TN) breast cancer and ovarian cancer susceptibility [3,41-43]. Interestingly, epidemiological and molecular evidence indicates that high-grade serous ovarian cancer arises not from ovarian epithelial cells, but from cells in the fimbriae of the fallopian tubes [44]. At any rate, the RAD51D alternative splicing profiles in fimbria, ovary and breast samples are similar [33], lending further support to the relevance of our analyses. As indicated, we have performed all experiments in malignant breast epithelial cell lines, despite the fact that the carcinogenic process may target the splicing machinery [45]. Yet, in a previous study, non-malignant cells (MCF-10A) demonstrated clear disadvantages such as very slow growth and high cell lethality after transfection [46]. Further, in the same study, we observed similar minigene splicing outcomes in malignant and non-malignant cell lines.

Many studies, including previous work by our group, support the ability of minigene assays to accurately reproduce splicing alterations as observed in RNA from carriers [16,24,25,47-49]. Unfortunately, as far as we know, none of the RAD51D variants here assessed have been tested previously in RNA from carriers (the only exception being a sub-optimal study performed in a c.738+1G $>$ A carrier, see footnote to Table 1). Yet, our RAD51D minigene (Figure 1) mimicked several physiological alternative splicing events [33], supporting the accuracy of the approach. Of note, splicing assays performed in RNA from carriers do not necessarily provide an accurate description of the actual spliceogenic effects caused by a genetic variant, as conflicting data in carriers of the same variant do exist (e.g., BRCA2 c.7976+5G>T or PALB2 c.3113+5G>C) [17,50-52]. In brief, caution should be taken when interpreting the biological and clinical implications of any splicing assay, irrespective of the experimental approach. Bearing this in mind, minigenes are certainly valuable tools for initial risk assessment, particularly if RNAs from carriers are not available.

In summary, we tested 53 RAD51D variants (37 from BRIDGES subjects and 16 artificial variants) in the $R A D 51 D$ minigene: 11 splice-site and 42 putative, SRE-disrupting variants. Forty-one of them (77.4\%; 11 splice-site and 30 ESE/ESS variants) impaired normal splicing patterns, supporting the high frequency of this deleterious mechanism. 


\subsection{Splice-Site Variants}

Historically, a splice variant was considered critical when it affected positions $\pm 1,2$, but other nucleotides are crucial for exon recognition, as well. Here, we analyzed 11 changes of the intron/exon boundaries, comprising seven $\pm 1,2$ variants, as well as other changes at intron positions $-3,-4,-8$ and +6 and the antepenultimate exon nucleotide. Our results showed that 10 variants dramatically disrupted splicing because they showed no trace, or residual amounts, of the FL transcript. Five variants disrupted the 3'SS, and remarkably, three of them induced the use of de novo or cryptic acceptor sites. Six variants disrupted or weakened the $5^{\prime}$ SS wherein exon skipping was the main outcome, although two of them also provoked the use of alternative donors. Only variant c. $263+6 \mathrm{~T}>\mathrm{C}$ showed a significant proportion of the FL transcript (49\%). Finally, it is worthy of mention that MES correctly anticipated disruptions of the canonical splice sites.

\subsection{SRE-Spliceogenic Variants}

Alternative splicing is a major mechanism for increasing gene expression complexity, producing multiple mRNA and protein isoforms, and is regulated by a huge array of factors [53]. RAD51D exon 3 is a non-constitutive exon [33], so it is conceivable that it is controlled by ESE/ESS elements [54]. Given the low accuracy of ESE/ESS predictors, we mapped exon 3 by overlapping exonic microdeletions. This approach revealed the existence of ESE motifs throughout this exon because all the microdeletions influenced exon 3 recognition, being particularly significant at interval c.202_235. This result confirmed that this region was characterized by a high density of cis-regulatory motifs that controlled exon 3 inclusion. Fine mapping using internal 10/12-bp deletions showed that interval c.210_219 was crucial in exon 3 recognition because its deletion strongly triggered exon skipping and did not show any trace of FL transcript. Consequently, potential spliceogenic ESE variants might have appeared in these ESE-rich segments. Altogether, we tested 42 potential ESE/ESS variants mapping to these ESE-rich intervals. Of note, in a region with high levels of naturally occurring alternative splicing and most variants having limited impacts, the definition of a spliceogenic variant is controversial. In this study, we defined an arbitrary $10 \%$ reduction cutoff for the contribution of the FL transcript to the overall expression (i.e., variants with $65.8 \%$ to $73.1 \%$ of the FL transcript were not considered spliceogenic). Based on this cut-off criteria, 30 variants (71.4\%; Table 2) impaired splicing, thus supporting our SRE-screening strategy. All spliceogenic SRE-variants but one (c.202G>A) produced non-negligible levels of FL transcripts (65.1\% to $27.4 \%$ ), with variants c.216C>G, c.215A>C, c.200_218del and c.187G >C producing $36.4 \%, 28.7 \%, 28.7 \%$ and $27.4 \%$, respectively. c. $202 \mathrm{G}>\mathrm{A}$ was the only tested SRE variant that did not generate FL transcripts. Curiously, c.214T>C presented the opposite effect, strengthening exon 3 inclusion to produce only FL transcripts.

The partial impact of these variants might have been due to the disruption of independent active ESEs, which, in physiological conditions, might synergistically cooperate for efficient exon recognition, as previously reported [27]. In effect, some deletions such as c.202_235del and c.200_219del (Table S4), which remove the crucial c.212_217 hotspot, display strong effects (no trace of the FL transcript) because they would simultaneously remove several active ESEs. In fact, any variant of the interval c.202_235 should be considered as potentially spliceogenic and functionally assayed. For instance, the recognition of atypical GC donors, which are usually associated with alternative splicing, requires the binding of several SR proteins such as SC35, Tra2 $\beta$, 9G8, SF2 / ASF and SRp40 [24,48,55]. Alternatively, there have been reported bi-functional SREs with enhancer and silencer properties such as the composite exonic splicing regulatory elements (CERES) [56,57], wherein the splicing impact is extremely difficult to predict. Exceptionally, one variant can decrease and increase in parallel the enhancer and silencer strengths, respectively, resulting in complete splicing anomalies. This is the case in the synonymous variant c.891C $>$ T of the SPINK5 gene (MIM \#605010), which induces exon 11 skipping. This change reduces the interaction with enhancing factor Tra $2 \beta$ and concurrently increases the binding of repressor 
hnRNPA1 [58]. Hence, RAD51D c.202G $>$ A, which did not generate any FL transcripts, in contrast to other close variants (i.e., c.198G $>$ T or c.208G $>$ A), might behave like this SPINK5 variant. Likewise, the spliceogenic variants that we report here might differently alter the ESE/ESS balance such that they would induce different impacts. Nevertheless, the participation of any activator/repressor splicing factors should be demonstrated by RNA binding assays.

With regard to the ESE/ESS prediction approaches, all of them showed advantages and disadvantages (Tables 2, S5 and S6). HEXplorer showed the highest sensitivity (74.1\%) and accuracy $(61.5 \%)$ but the lowest specificity (33.3\%); in fact, HEXplorer would have selected most variants of this critical segment (28 variants). However, the estimations of HSF, HEXplorer, Hot-Skip and $\triangle$ tESRseq would have failed in detecting 19, 7, 19 and 17 spliceogenic variants, respectively. For example, c.184T $>$ A, which presented a moderately strong impact ( $62.8 \%$ of abnormal transcripts), would only have been detected by the less sensitive program (HSF, 29.6\%). Tubeuf et al. [59] evaluated four ESE/ESS predictors, including $\triangle \mathrm{tESRseq}$ and HEXplorer, in a large dataset of $>1300$ variants, wherein they found greater accuracy of both tools, HEXplorer specificity and $\Delta$ tESRseq sensitivity than in our study. This may be due to the small size of our sample or specific exon/gene parameters that may affect the performance of SRE predictors, as indicated by these authors. Nevertheless, the low accuracy of ESE/ESS prediction tools lays bare the need to improve the detection algorithms. The cumulative knowledge of the splicing regulation and functional tests could provide a basis for increasing the precision of SRE algorithms and the prediction of variant impacts. On the other hand, ESE functional mapping by exonic microdeletions appears as an optimal and reasonable strategy for discovering SRE hotspots $[25,27]$. Consequently, we propose to test all the reported variants that are located in these specific, highly spliceogenic intervals. Furthermore, the identification of spliceogenic ESE variants should be focused on exons that are targeted by prevalent alternative events.

\subsection{ACMG/AMP-Based Classification of Spliceogenic Variants}

Because RAD51D expert panel specifications of the ACMG/AMP guidelines were not yet available (clinicalgenome.org/, last accessed on 29 December 2020), we developed our own tentative specifications (Supplementary Methods). Upon doing that, we faced several challenges, including: (i) combining predictive and functional splicing evidence, (ii) assigning specific PS3 / BS3 code strengths to complex readouts (i.e., two or more mRNA transcripts with different predicted impacts on the coding sequence), and (iii) integrating a high level of naturally occurring alternative splicing into the classification system, which is of particular relevance when evaluating the pathogenicity of VUS [60]. As far as we know, no specific ClinGen-SVI recommendations addressing these issues have been yet released [31].

We tackled them by incorporating into the classification system some ad hoc rules based on structural/functional predictions and expert judgment (e.g., when assigning a PS3 code strength to complex splicing readouts, we ignored transcripts representing $<10 \%$ of the overall expression). Therefore, we do not intend to provide here (see Table 3) a definitive clinical classification of genetic variants, but rather to highlight some limitations of the current ACMG/AMP framework to interpret complex spliceogenic outcomes (as in those observed for several RAD51D variants, see Tables 1 and 2).

However, we think that, overall, our approach produces meaningful classifications, with spliceogenic variants ending up as variants of uncertain significance if expressing either a significant proportion of full-length transcripts (e.g., c.263+6T>C, c. $185 \mathrm{C}>\mathrm{T}$ ) or in-frame transcripts of unknown impact on protein function (e.g., c.476_480+1dup, c. $481-8 \mathrm{C}>\mathrm{A})$.

RAD51D c.202G $>\mathrm{A}$ is remarkable in that, contrary to most exon 3 variants, full-length transcripts are not produced. Yet, we have ended up classifying this variant as of uncertain significance (rather than likely pathogenic) based on: (i) the minigene readout showing a 
significant proportion of in-frame $\Delta$ (E3p36) transcripts, and (ii) the variant being detected in control samples (Table 3). Interestingly, $R A D 51 D$ c.214T $>C$ produced only full-length transcripts (therefore, suppressing the level of alternative splicing observed in wt alleles). The clinical relevance, if any, of suppressing naturally occurring alternative splicing, is far from obvious. At any rate, canonical transcripts code for a rare missense change (p.Tyr72His) that warrants classification as of uncertain significance. Indeed, for this and other RAD51D variants predicted to introduce missense changes and/or in-frame alterations, additional analyses are required to assess consequences for gene function.

Current ACMG/AMP guidelines [29] have not been developed to identify "intermediate risk variants". Yet, we think it is worth considering this possibility for certain variants expressing variable proportions of (likely) functional and (likely) non-functional mRNAs (see Table 3 and Supplementary Methods). It will be extremely challenging to evaluate risk for individual $R A D 51 D$ variants that are exceedingly rare in the population, but it should be possible, in principle, to design burden analyses for variants displaying similar splicing effects.

\section{Conclusions}

We demonstrated through minigene-based analysis that splicing disruptions were induced by 28 out of $37 R A D 51 D$ variants $(75.7 \%)$ originally identified in breast cancer cases and/or healthy controls of the BRIDGES cohort. Functional mapping by exonic microdeletions was a reliable strategy to identify SRE variants that impaired splicing. Indeed, using this approach, we identified RAD51D exon 3 sequences in which any genetic variant (regardless of its predicted coding impact) was a candidate spliceogenic variant. Finally, we have shown that current ACMG/AMP guidelines raise several issues when applied to variants associated with complex spliceogenic alterations.

Supplementary Materials: The following are available online at https:/ /www.mdpi.com/article/10 .3390 / cancers13112845/s1, Figure S1: Workflow of the minigene protocol, Figure S2: Insert sequence of minigene mgR51D_ex2-9, Figure S3: Agarose gel (1.0\%) electrophoresis of the splicing assays of eight splice-site variants in MDA-MB-231 and MCF-7 cells, Figure S4: Schematic representation of the most common aberrant transcripts, Figure S5: Fluorescent fragment analysis of other exon 3 microdeletions and variants, Figure S6: Mapping human RAD51D regions critical to protein function, Figure S7: Mapping of 9 RAD51D C-terminal $\beta$-strands, Figure S8: PS3/BS3 annotation of RAD51D-altered and canonical transcripts, Table S1: Mutagenesis primers for RAD51D variants and microdeletions, Table S2: Bioinformatics analysis of RAD51D variants with Max Ent Score, Table S3: RNA and protein HGVS annotations according to transcript ENST00000345365.10, Table S4: Functional mapping of ESEs by exonic microdeletions, Table S5: Accuracy of bioinformatics predictions, Table S6: Sensitivity and specificity of the splicing programs, Supplementary Methods: ACMG/AMP-like classification of 37 RAD51D variants based on PS3/BS3 functional evidence. References [61-71] are cited in Supplementary Materials.

Author Contributions: Conceptualization, M.d.l.H. and E.A.V.; data curation, E.B.-M., L.S.-M., A.V.P., S.C., J.A., D.F.E., P.D. and M.P.G.V.; formal analysis, E.B.-M., L.S.-M., A.V.-P., M.d.l.H. and E.A.V.; funding acquisition, P.D., D.F.E., M.P.G.V., M.d.l.H. and E.A.V.; investigation, E.B.-M., L.S.-M., A.V.-P., V.L., M.I., C.L., P.P.-S., A.G.-S., M.P.G.V., M.d.l.H. and E.A.V.; methodology, A.V.-P., L.S.-M., E.B.-M., M.I. and E.A.V.; supervision, E.A.V.; writing—original draft, E.B.-M., M.d.l.H. and E.A.V.; writingreview and editing, E.B.-M., M.P.G.V., M.d.l.H. and E.A.V. All authors have read and agreed to the published version of the manuscript.

Funding: P.D., M.P.G.V., D.F.E., M.d.l.H. and E.A.V. have received funding from the European Union's Horizon 2020 research and innovation program under grant agreement no. 634935. E.A.V.'s lab is supported by grants from the Spanish Ministry of Science and Innovation, Plan Nacional de I + D + I 2013-2016, ISCIII (PI17/00227 and PI20/00225), co-funded by FEDER from Regional Development European Funds (European Union) and from the Consejería de Educación, Junta de Castilla y León, ref. CSI242P18 (actuación cofinanciada P.O. FEDER 2014-2020 de Castilla y León). M.d.l.H.'s lab is supported by grants from the Spanish Ministry of Science and Innovation, Plan Nacional de I + D + I 2013-2016, ISCIII (PI15/00059 and PI20/00110) and co-funded by FEDER from 
Regional Development European Funds (European Union). L.S.-M. is supported by a predoctoral fellowship from the AECC-Scientific Foundation, Sede Provincial de Valladolid (2019-2023). A.V.-P. is supported by a predoctoral fellowship from the Consejería de Educación, Junta de Castilla y León (2018-2022). Programa Estratégico Instituto de Biología y Genética Molecular (IBGM), Escalera de Excelencia, Junta de Castilla y León (Ref. CLU-2019-02).

Institutional Review Board Statement: The study was conducted according to the guidelines of the Declaration of Helsinki, and approved by the Institutional Review Board (or Ethics Committee) of the Spanish National Research Council (CSIC) (date of approval: 28 May 2018).

Informed Consent Statement: Not applicable.

Data Availability Statement: After publication, all sequencing and fragment analysis data will be available at Figshare (DOI: 10.6084/m9.figshare.10272029).

Acknowledgments: We thank Alicia García Álvarez (IBGM-CSIC) for her technical support.

Conflicts of Interest: The authors declare no conflict of interest.

\section{References}

1. Sullivan, M.R.; Bernstein, K.A. RAD-ical New Insights into RAD51 Regulation. Genes 2018, 9, 629. [CrossRef] [PubMed]

2. Scully, R.; Panday, A.; Elango, R.; Willis, N.A. DNA double-strand break repair-pathway choice in somatic mammalian cells. Nat. Rev. Mol. Cell Biol. 2019, 20, 698-714. [CrossRef]

3. Yang, X.; Song, H.; Leslie, G.; Engel, C.; Hahnen, E.; Auber, B.; Horváth, J.; Kast, K.; Niederacher, D.; Turnbull, C.; et al. Ovarian and breast cancer risks associated with pathogenic variants in RAD51C and RAD51D. J. Natl. Cancer Inst. 2020, 37, 1-9. [CrossRef]

4. Loveday, C.; Turnbull, C.; Ramsay, E.; Hughes, D.; Ruark, E.; Frankum, J.R.; Bowden, G.; Kalmyrzaev, B.; Warren-Perry, M.; Snape, K.; et al. Germline mutations in RAD51D confer susceptibility to ovarian cancer. Nat. Genet. 2011, 43, 879-882. [CrossRef] [PubMed]

5. Dorling, L.; Carvalho, S.; Allen, J.; González-Neira, A.; Luccarini, C.; Wahlström, C.; Pooley, K.A.; Parsons, M.T.; Fortuno, C.; Wang, Q.; et al. Breast Cancer Risk Genes-Association Analysis in More than 113,000 Women. N. Engl. J. Med. 2021, 384, 428-439.

6. Hu, C.; Hart, S.N.; Gnanaolivu, R.; Huang, H.; Lee, K.Y.; Na, J.; Gao, C.; Lilyquist, J.; Yadav, S.; Boddicker, N.J.; et al. A Population-Based Study of Genes Previously Implicated in Breast Cancer. N. Engl. J. Med. 2021, 384, 440-451. [CrossRef] [PubMed]

7. Manning, K.S.; Cooper, T.A. The roles of RNA processing in translating genotype to phenotype. Nat. Rev. Mol. Cell Biol. 2017, 18, 102-114. [CrossRef] [PubMed]

8. Scotti, M.M.; Swanson, M.S. RNA mis-splicing in disease. Nat. Rev. Genet. 2016, 17, 19-32. [CrossRef]

9. Lopez-Bigas, N.; Audit, B.; Ouzounis, C.; Parra, G.; Guigo, R.; López-Bigas, N.; Audit, B.; Ouzounis, C.; Parra, G.; Guigó, R. Are splicing mutations the most frequent cause of hereditary disease? FEBS Lett. 2005, 579, 1900-1903. [CrossRef] [PubMed]

10. Velázquez, C.; De Leeneer, K.; Esteban-Cardeñosa, E.M.; Avila Cobos, F.; Lastra, E.; Abella, L.E.; de la Cruz, V.; Lobatón, C.D.; Claes, K.B.; Durán, M.; et al. Germline Genetic Findings Which May Impact Therapeutic Decisions in Families with a Presumed Predisposition for Hereditary Breast and Ovarian Cancer. Cancers 2020, 12, 2151. [CrossRef]

11. Gelli, E.; Colombo, M.; Pinto, A.; De Vecchi, G.; Foglia, C.; Amitrano, S.; Morbidoni, V.; Imperatore, V.; Manoukian, S.; Baldassarri, M.; et al. Usefulness and Limitations of Comprehensive Characterization of mRNA Splicing Profiles in the Definition of the Clinical Relevance of BRCA1/2 Variants of Uncertain Significance. Cancers 2019, 11, 295. [CrossRef] [PubMed]

12. Morbidoni, V.; Baschiera, E.; Forzan, M.; Fumini, V.; Ali, D.S.; Giorgi, G.; Buson, L.; Desbats, M.A.; Cassina, M.; Clementi, M.; et al. Hybrid Minigene Assay: An Efficient Tool to Characterize mRNA Splicing Profiles of NF1 Variants. Cancers 2021, 13, 999. [CrossRef] [PubMed]

13. Bonnet, C.; Krieger, S.; Vezain, M.; Rousselin, A.; Tournier, I.; Martins, A.; Berthet, P.; Chevrier, A.; Dugast, C.; Layet, V.; et al. Screening BRCA1 and BRCA2 unclassified variants for splicing mutations using reverse transcription PCR on patient RNA and an ex vivo assay based on a splicing reporter minigene. J. Med. Genet. 2008, 45, 438-446. [CrossRef]

14. Whiley, P.J.; de la Hoya, M.; Thomassen, M.; Becker, A.; Brandão, R.; Pedersen, I.S.; Montagna, M.; Menéndez, M.; Quiles, F.; Gutiérrez-Enríquez, S.; et al. Comparison of mRNA splicing assay protocols across multiple laboratories: Recommendations for best practice in standardized clinical testing. Clin. Chem. 2014, 60, 341-352. [CrossRef]

15. Caputo, S.M.; Léone, M.; Damiola, F.; Ehlen, A.; Carreira, A.; Gaidrat, P.; Martins, A.; Brandão, R.D.; Peixoto, A.; Vega, A.; et al. Full in-frame exon 3 skipping of BRCA2 confers high risk of breast and/or ovarian cancer. Oncotarget 2018, 9, 17334-17348. [CrossRef]

16. Sanoguera-Miralles, L.; Valenzuela-Palomo, A.; Bueno-Martínez, E.; Llovet, P.; Díez-Gómez, B.; Caloca, M.J.; Pérez-Segura, P.; Fraile-Bethencourt, E.; Colmena, M.; Carvalho, S.; et al. Comprehensive Functional Characterization and Clinical Interpretation of 20 Splice-Site Variants of the RAD51C Gene. Cancers 2020, 12, 3771. [CrossRef] 
17. Lopez-Perolio, I.; Leman, R.; Behar, R.; Lattimore, V.; Pearson, J.F.; Castéra, L.; Martins, A.; Vaur, D.; Goardon, N.; Davy, G.; et al. Alternative splicing and ACMG-AMP-2015-based classification of PALB2 genetic variants: An ENIGMA report. J. Med. Genet. 2019, 56, 453-460. [CrossRef]

18. Yeo, G.; Burge, C.B. Maximum entropy modeling of short sequence motifs with applications to RNA splicing signals. J. Comput. Biol. 2004, 11, 377-394. [CrossRef]

19. Reese, M.G.; Eeckman, F.H.; Kulp, D.; Haussler, D. Improved splice site detection in Genie. J. Comput. Biol. 1997, 4, 311-323. [CrossRef] [PubMed]

20. Houdayer, C.; Caux-Moncoutier, V.; Krieger, S.; Barrois, M.; Bonnet, F.; Bourdon, V.; Bronner, M.; Buisson, M.; Coulet, F.; Gaildrat, P.; et al. Guidelines for splicing analysis in molecular diagnosis derived from a set of 327 combined in silico/in vitro studies on BRCA1 and BRCA2 variants. Hum. Mutat. 2012, 33, 1228-1238. [CrossRef]

21. Erkelenz, S.; Theiss, S.; Otte, M.; Widera, M.; Peter, J.O.; Schaal, H. Genomic HEXploring allows landscaping of novel potential splicing regulatory elements. Nucleic Acids Res. 2014, 42, 10681-10697. [CrossRef] [PubMed]

22. Raponi, M.; Kralovicova, J.; Copson, E.; Divina, P.; Eccles, D.; Johnson, P.; Baralle, D.; Vorechovsky, I. Prediction of singlenucleotide substitutions that result in exon skipping: Identification of a splicing silencer in BRCA1 exon 6. Hum. Mutat. 2011, 32, 436-444. [CrossRef] [PubMed]

23. Ke, S.; Shang, S.; Kalachikov, S.M.; Morozova, I.; Yu, L.; Russo, J.J.; Ju, J.; Chasin, L.A. Quantitative evaluation of all hexamers as exonic splicing elements. Genome Res. 2011, 21, 1360-1374. [CrossRef] [PubMed]

24. Fraile-Bethencourt, E.; Díez-Gómez, B.; Velásquez-Zapata, V.; Acedo, A.; Sanz, D.J.; Velasco, E.A. Functional classification of DNA variants by hybrid minigenes: Identification of 30 spliceogenic variants of BRCA2 exons 17 and 18. PLoS Genet. 2017, 13, e1006691. [CrossRef] [PubMed]

25. Acedo, A.; Hernández-Moro, C.; Curiel-García, Á.; Díez-Gómez, B.; Velasco, E.A. Functional classification of BRCA2 DNA variants by splicing assays in a large minigene with 9 exons. Hum. Mutat. 2015, 36, 210-221. [CrossRef]

26. De Garibay, G.R.; Acedo, A.; García-Casado, Z.; Gutiérrez-Enríquez, S.; Tosar, A.; Romero, A.; Garre, P.; Llort, G.; Thomassen, M.; Díez, O.; et al. Capillary electrophoresis analysis of conventional splicing assays: IARC analytical and clinical classification of 31 BRCA2 genetic variants. Hum. Mutat. 2014, 35, 53-57. [CrossRef]

27. Acedo, A.; Sanz, D.J.; Durán, M.; Infante, M.; Pérez-Cabornero, L.; Miner, C.; Velasco, E.A. Comprehensive splicing functional analysis of DNA variants of the BRCA2 gene by hybrid minigenes. Breast Cancer Res. 2012, 14, R87. [CrossRef]

28. Montalban, G.; Bonache, S.; Moles-Fernández, A.; Gadea, N.; Tenés, A.; Torres-Esquius, S.; Carrasco, E.; Balmaña, J.; Diez, O.; Gutiérrez-Enríquez, S. Incorporation of semi-quantitative analysis of splicing alterations for the clinical interpretation of variants in BRCA1 and BRCA2 genes. Hum. Mutat. 2019, 40, 2296-2317. [CrossRef]

29. Richards, S.; Aziz, N.; Bale, S.; Bick, D.; Das, S.; Gastier-Foster, J.; Grody, W.W.; Hegde, M.; Lyon, E.; Spector, E.; et al. Standards and guidelines for the interpretation of sequence variants: A joint consensus recommendation of the American College of Medical Genetics and Genomics and the Association for Molecular Pathology. Genet. Med. 2015, 17, 405-424. [CrossRef]

30. Abou Tayoun, A.N.; Pesaran, T.; DiStefano, M.T.; Oza, A.; Rehm, H.L.; Biesecker, L.G.; Harrison, S.M.; ClinGen Sequence Variant Interpretation Working Group (ClinGen SVI). Recommendations for interpreting the loss of function PVS1 ACMG/AMP variant criterion. Hum. Mutat. 2018, 39, 1517-1524. [CrossRef]

31. Brnich, S.E.; Abou Tayoun, A.N.; Couch, F.J.; Cutting, G.R.; Greenblatt, M.S.; Heinen, C.D.; Kanavy, D.M.; Luo, X.; McNulty, S.M.; Starita, L.M.; et al. Recommendations for application of the functional evidence PS3/BS3 criterion using the ACMG/AMP sequence variant interpretation framework. Genome Med. 2020, 12, 3. [CrossRef] [PubMed]

32. Lee, K.; Krempely, K.; Roberts, M.E.; Anderson, M.J.; Carneiro, F.; Chao, E.; Dixon, K.; Figueiredo, J.; Ghosh, R.; Huntsman, D.; et al. Specifications of the ACMG/AMP variant curation guidelines for the analysis of germline CDH1 sequence variants. Hum. Mutat. 2018, 39, 1553-1568. [CrossRef] [PubMed]

33. Brandão, R.D.; Mensaert, K.; López-Perolio, I.; Tserpelis, D.; Xenakis, M.; Lattimore, V.; Walker, L.C.; Kvist, A.; Vega, A.; Gutiérrez-Enríquez, S.; et al. Targeted RNA-seq successfully identifies normal and pathogenic splicing events in breast/ovarian cancer susceptibility and Lynch syndrome genes. Int. J. Cancer 2019, 145, 401-414. [CrossRef] [PubMed]

34. Fairbrother, W.G.; Holste, D.; Burge, C.B.; Sharp, P.A. Single nucleotide polymorphism-based validation of exonic splicing enhancers. PLoS Biol. 2004, 2, E268. [CrossRef] [PubMed]

35. Keren, H.; Lev-Maor, G.; Ast, G. Alternative splicing and evolution: Diversification, exon definition and function. Nat. Rev. Genet. 2010, 11, 345-355. [CrossRef]

36. Canson, D.; Glubb, D.; Spurdle, A.B. Variant effect on splicing regulatory elements, branchpoint usage, and pseudoexonization: Strategies to enhance bioinformatic prediction using hereditary cancer genes as exemplars. Hum. Mutat. 2020, 41, $1705-1721$. [CrossRef]

37. Soukarieh, O.; Gaildrat, P.; Hamieh, M.; Drouet, A.; Baert-Desurmont, S.; Frébourg, T.; Tosi, M.; Martins, A. Exonic Splicing Mutations Are More Prevalent than Currently Estimated and Can Be Predicted by Using In Silico Tools. PLoS Genet. 2016, 12, e1005756.

38. Konstanta, I.; Fostira, F.; Apostolou, P.; Stratikos, E.; Kalfakakou, D.; Pampanos, A.; Kollia, P.; Papadimitriou, C.; Konstantopoulou, I.; Yannoukakos, D. Contribution of RAD51D germline mutations in breast and ovarian cancer in Greece. J. Hum. Genet. 2018, 63, 1149-1158. [CrossRef] [PubMed] 
39. Van Marcke, C.; Collard, A.; Vikkula, M.; Duhoux, F.P. Prevalence of pathogenic variants and variants of unknown significance in patients at high risk of breast cancer: A systematic review and meta-analysis of gene-panel data. Crit. Rev. Oncol. Hematol. 2018, 132, 138-144. [CrossRef] [PubMed]

40. Rhine, C.L.; Neil, C.; Glidden, D.T.; Cygan, K.J.; Fredericks, A.M.; Wang, J.; Walton, N.A.; Fairbrother, W.G. Future directions for high-throughput splicing assays in precision medicine. Hum. Mutat. 2019, 40, 1225-1234. [CrossRef]

41. Alenezi, W.M.; Fierheller, C.T.; Recio, N.; Tonin, P.N. Literature review of BARD1 as a cancer predisposing gene with a focus on breast and ovarian cancers. Genes 2020, 11, 856. [CrossRef]

42. Norquist, B.M.; Harrell, M.I.; Brady, M.F.; Walsh, T.; Lee, M.K.; Gulsuner, S.; Bernards, S.S.; Casadei, S.; Yi, Q.; Burger, R.A.; et al. Inherited mutations in women with ovarian carcinoma. JAMA Oncol. 2016, 2, 482-490. [CrossRef] [PubMed]

43. Pavanello, M.; Chan, I.H.Y.; Ariff, A.; Pharoah, P.D.P.; Gayther, S.A.; Ramus, S.J. Rare germline genetic variants and the risks of epithelial ovarian cancer. Cancers 2020, 12, 3046. [CrossRef] [PubMed]

44. Kyo, S.; Ishikawa, N.; Nakamura, K.; Nakayama, K. The fallopian tube as origin of ovarian cancer: Change of diagnostic and preventive strategies. Cancer Med. 2020, 9, 421-431. [CrossRef] [PubMed]

45. Kim, E.; Goren, A.; Ast, G. Insights into the connection between cancer and alternative splicing. Trends Genet. 2008, 24, 7-10. [CrossRef] [PubMed]

46. Sanz, D.J.; Acedo, A.; Infante, M.; Durán, M.; Pérez-Cabornero, L.; Esteban-Cardeñosa, E.; Lastra, E.; Pagani, F.; Miner, C.; Velasco, E.A. A high proportion of DNA variants of BRCA1 and BRCA2 is associated with aberrant splicing in breast/ovarian cancer patients. Clin. Cancer Res. 2010, 16, 1957-1967. [CrossRef] [PubMed]

47. Fraile-Bethencourt, E.; Valenzuela-Palomo, A.; Díez-Gómez, B.; Acedo, A.; Velasco, E.A. Identification of Eight Spliceogenic Variants in BRCA2 Exon 16 by Minigene Assays. Front. Genet. 2018, 9, 188. [CrossRef]

48. Fraile-Bethencourt, E.; Valenzuela-Palomo, A.; Díez-Gómez, B.; Goina, E.; Acedo, A.; Buratti, E.; Velasco, E.A. Mis-splicing in breast cancer: Identification of pathogenic BRCA2 variants by systematic minigene assays. J. Pathol. 2019, 248, 409-420. [CrossRef]

49. Fraile-Bethencourt, E.; Valenzuela-Palomo, A.; Díez-Gómez, B.; Caloca, M.J.; Gómez-Barrero, S.; Velasco, E.A. Minigene Splicing Assays Identify 12 Spliceogenic Variants of BRCA2 Exons 14 and 15. Front. Genet. 2019, 10, 503. [CrossRef]

50. Reid, S.; Schindler, D.; Hanenberg, H.; Barker, K.; Hanks, S.; Kalb, R.; Neveling, K.; Kelly, P.; Seal, S.; Freund, M.; et al. Biallelic mutations in PALB2 cause Fanconi anemia subtype FA-N and predispose to childhood cancer. Nat. Genet. 2007, 39, 162-164. [CrossRef]

51. Montalban, G.; Fraile-Bethencourt, E.; López-Perolio, I.; Pérez-Segura, P.; Infante, M.; Durán, M.; Alonso-Cerezo, M.C.; LópezFernández, A.; Diez, O.; de la Hoya, M.; et al. Characterization of spliceogenic variants located in regions linked to high levels of alternative splicing: BRCA2 c.7976+5G>T as a case study. Hum. Mutat. 2018, 39, 1155-1160. [CrossRef]

52. Landrith, T.; Li, B.; Cass, A.A.; Conner, B.R.; LaDuca, H.; McKenna, D.B.; Maxwell, K.N.; Domchek, S.; Morman, N.A.; Heinlen, C.; et al. Splicing profile by capture RNA-seq identifies pathogenic germline variants in tumor suppressor genes. NPJ Precis. Oncol. 2020, 4, 4. [CrossRef]

53. Cáceres, J.F.; Kornblihtt, A.R. Alternative splicing: Multiple control mechanisms and involvement in human disease. Trends Genet. 2002, 18, 186-193. [CrossRef]

54. Goina, E.; Skoko, N.; Pagani, F. Binding of DAZAP1 and hnRNPA1/A2 to an Exonic Splicing Silencer in a Natural BRCA1 Exon 18 Mutant. Mol. Cell. Biol. 2008, 28, 3850-3860. [CrossRef] [PubMed]

55. Kralovicova, J.; Hwang, G.; Asplund, A.C.; Churbanov, A.; Smith, C.I.E.; Vorechovsky, I. Compensatory signals associated with the activation of human GC 5' splice sites. Nucleic Acids Res. 2011, 39, 7077-7091. [CrossRef] [PubMed]

56. Haque, A.; Buratti, E.; Baralle, F.E. Functional properties and evolutionary splicing constraints on a composite exonic regulatory element of splicing in CFTR exon 12. Nucleic Acids Res. 2010, 38, 647-659. [CrossRef] [PubMed]

57. Pagani, F.; Stuani, C.; Tzetis, M.; Kanavakis, E.; Efthymiadou, A.; Doudounakis, S.; Casals, T.; Baralle, F.E. New type of disease causing mutations: The example of the composite exonic regulatory elements of splicing in CFTR exon 12. Hum. Mol. Genet. 2003, 12, 1111-1120. [CrossRef] [PubMed]

58. Dal Mas, A.; Fortugno, P.; Donadon, I.; Levati, L.; Castiglia, D.; Pagani, F. Exon-Specific U1s Correct SPINK 5 Exon 11 Skipping Caused by a Synonymous Substitution that Affects a Bifunctional Splicing Regulatory Element. Hum. Mutat. 2015, 36, 504-512. [CrossRef]

59. Tubeuf, H.; Charbonnier, C.; Soukarieh, O.; Blavier, A.; Lefebvre, A.; Dauchel, H.; Frebourg, T.; Gaildrat, P.; Martins, A. Large-scale comparative evaluation of user-friendly tools for predicting variant-induced alterations of splicing regulatory elements. Hum. Mutat. 2020, 41, 1811-1829. [CrossRef]

60. Dosil, V.; Tosar, A.; Cañadas, C.; Pérez-Segura, P.; Díaz-Rubio, E.; Caldés, T.; de la Hoya, M. Alternative splicing and molecular characterization of splice site variants: BRCA1 c.591C>T as a case study. Clin. Chem. 2010, 56, 53-61. [CrossRef]

61. Kim, Y.M.; Choi, B.-S. Structural and functional characterization of the N-terminal domain of human Rad51D. Int. J. Biochem. Cell Biol. 2011, 43, 416-422. [CrossRef]

62. Miller, K.A. Domain mapping of the Rad51 paralog protein complexes. Nucleic Acids Res. 2004, 32, 169-178. [CrossRef]

63. Shin, D.S.; Pellegrini, L.; Daniels, D.; Yelent, B.; Craig, L.; Bates, D.; Yu, D.S.; Shivji, M.K.; Hitomi, C.; Arvai, A.S.; et al. Full-length archaeal Rad51 structure and mutants: Mechanisms for RAD51 assembly and control by BRCA2. EMBO J. 2003, 22, $4566-4576$. [CrossRef] 
64. Chang, Y.-W.; Ko, T.-P.; Lee, C.-D.; Chang, Y.-C.; Lin, K.-A.; Chang, C.-S.; Wang, A.H.-J.; Wang, T.-F. Three New Structures of Left-Handed RadA Helical Filaments: Structural Flexibility of N-Terminal Domain Is Critical for Recombinase Activity. PLoS ONE 2009, 4, e4890. [CrossRef]

65. Tavtigian, S.V.; on behalf of the ClinGen Sequence Variant Interpretation Working Group (ClinGen SVI); Greenblatt, M.S.; Harrison, S.M.; Nussbaum, R.L.; Prabhu, S.A.; Boucher, K.M.; Biesecker, L.G. Modeling the ACMG/AMP variant classification guidelines as a Bayesian classification framework. Genet. Med. 2018, 20, 1054-1060. [CrossRef]

66. Baldock, R.A.; Pressimone, C.A.; Baird, J.M.; Khodakov, A.; Luong, T.; Grundy, M.K.; Smith, C.M.; Karpenshif, Y.; Bratton-Palmer, D.S.; Prakash, R.; et al. RAD51D splice variants and cancer-associated mutations reveal XRCC2 interaction to be critical for homologous recombination. DNA Repair 2019, 76, 99-107. [CrossRef] [PubMed]

67. Gruver, A.M.; Miller, K.A.; Rajesh, C.; Smiraldo, P.G.; Kaliyaperumal, S.; Balder, R.; Stiles, K.M.; Albala, J.S.; Pittman, D.L. The ATPase motif in RAD51D is required for resistance to DNA interstrand crosslinking agents and interaction with RAD51C. Mutagenesis 2005, 20, 433-440. [CrossRef] [PubMed]

68. Wiese, C.; Hinz, J.M.; Tebbs, R.S.; Nham, P.B.; Urbin, S.S.; Collins, D.W.; Thompson, L.H.; Schild, D. Disparate requirements for the Walker A and B ATPase motifs of human RAD51D in homologous recombination. Nucleic Acids Res. 2006, 34, $2833-2843$. [CrossRef] [PubMed]

69. Whiffin, N.; Minikel, E.; Walsh, R.; O’Donnell-Luria, A.H.; Karczewski, K.; Ing, A.Y.; Barton, P.J.R.; Funke, B.; A Cook, S.; MacArthur, D.; et al. Using high-resolution variant frequencies to empower clinical genome interpretation. Genet. Med. 2017, 19, 1151-1158. [CrossRef] [PubMed]

70. Hu, C.; Polley, E.C.; Yadav, S.; Lilyquist, J.; Shimelis, H.; Na, J.; Hart, S.N.; E Goldgar, D.; Shah, S.; Pesaran, T.; et al. The Contribution of Germline Predisposition Gene Mutations to Clinical Subtypes of Invasive Breast Cancer From a Clinical Genetic Testing Cohort. J. Natl. Cancer Inst. 2020, 112, 1231-1241. [CrossRef] [PubMed]

71. Lilyquist, J.; LaDuca, H.; Polley, E.; Davis, B.T.; Shimelis, H.; Hu, C.; Hart, S.N.; Dolinsky, J.S.; Couch, F.J.; Goldgar, D.E. Frequency of mutations in a large series of clinically ascertained ovarian cancer cases tested on multi-gene panels compared to reference controls. Gynecol. Oncol. 2017, 147, 375-380. [CrossRef] [PubMed] 